\title{
Transient Exciplex Formation Electron Transfer Mechanism
}

\author{
Michael G. Kuzmin, Irina V. Soboleva, and Elena V. Dolotova \\ Department of Chemistry, M.V. Lomonosov Moscow State University, Moscow 119992, Russia \\ Correspondence should be addressed to Michael G. Kuzmin, kuzmin@photo.chem.msu.ru
}

Received 27 May 2011; Accepted 13 September 2011

Academic Editor: James McCusker

Copyright ( 12011 Michael G. Kuzmin et al. This is an open access article distributed under the Creative Commons Attribution License, which permits unrestricted use, distribution, and reproduction in any medium, provided the original work is properly cited.

\begin{abstract}
Transient exciplex formation mechanism of excited-state electron transfer reactions is analyzed in terms of experimental data on thermodynamics and kinetics of exciplex formation and decay. Experimental profiles of free energy, enthalpy, and entropy for transient exciplex formation and decay are considered for several electron transfer reactions in various solvents. Strong electronic coupling in contact pairs of reactants causes substantial decrease of activation energy relative to that for conventional long-range ET mechanism, especially for endergonic reactions, and provides the possibility for medium reorganization concatenated to gradual charge shift in contrast to conventional preliminary medium and reactants reorganization. Experimental criteria for transient exciplex formation (concatenated) mechanism of excited-state electron transfer are considered. Available experimental data show that this mechanism dominates for endergonic ET reactions and provides a natural explanation for a lot of known paradoxes of ET reactions.
\end{abstract}

\section{Introduction}

Electron transfer (ET) reactions are known to proceed at different distances between reactant molecules in loose reactant pairs (long range ET or outer sphere reaction) as well as in tight pairs (contact ET or inner sphere reaction) [1-4]. Conventional approaches to long-range ET are Marcus theory $[1,2,5,6]$ and radiationless transitions theory [7-9]. They consider excited-state ET as a transition between two potential minima-from initial locally excited electronic state (LE) into charge transfer (CT) state either by preliminary thermally activated reorganization of the medium and reactants (necessary for degeneration of electronic levels in the molecules of the reactants and products) or by radiationless quantum transition which requires no preliminary activation and occurs in exergonic region (when $\Delta G_{\mathrm{ET}}<-\lambda$, where $\lambda$ is the reorganization energy). Frequently both these theories are considered in terms of unified approach $[3,4,6]$ (since $\Delta E=$ $-\left(\Delta G_{\mathrm{ET}}{ }^{*}+\lambda\right)$, and parabolic terms used in the Marcus theory and harmonic approximation used in radiationless transitions theory provide very similar mathematical expressions in spite of different physical behavior of these mechanisms).
In tight pairs strong electronic coupling of LE and CT states $\left(r_{\mathrm{AD}}<0.6 \mathrm{~nm}, V_{\mathrm{AD}}>0.1 \mathrm{eV}\right)$ can cause an appearance of common potential minimum (Figure 1). In this case an intermediate with partial charge transfer $\left(\mathrm{A}^{-z} \mathrm{D}^{+z}, 0<\right.$ $z<1$ ) is formed, and the reaction mechanism is no longer that of a single elementary step through a transition state. In excited-state ET reactions these transients were observed experimentally and are well known as exciplexes [10-17]. Strong electronic coupling can even cause some decrease of the diffusion barrier for formation of contact pair from loose pair of reactants. Formation of exciplexes in some excited-state reaction between electron donors and acceptors was observed experimentally much earlier by their emission and absorption spectra [18-22] but their role in total ET reactions mechanism was realized only in 90 s.

There are two important features that distinguish this mechanism: (1) small energy barrier for charge shift between the reactants; (2) efficient radiationless decay in the transient exciplex, which competes with the formation of final products (radical ions), caused by relatively long lifetime of transient exciplexes $\left(\sim 10^{-8}-10^{-11} \mathrm{~s}\right)$ in contrast to the transition state $\left(\sim 10^{-13}-10^{-15} \mathrm{~s}\right)$. This radiationless decay in transients can decrease the products quantum yield substantially [23-29]. 




Figure 1: (Top) Dependences of the free energy on the medium and reactants reorganization coordinate $s$ for isoergonic ET reaction for different distances between reactant molecules: $r_{\mathrm{AD}}=0.35 \mathrm{~nm}$, $V_{\mathrm{AD}}=0.22 \mathrm{eV}(1), r_{\mathrm{AD}}=0.5 \mathrm{~nm}, V_{\mathrm{AD}}=0.1 \mathrm{eV}(2), r_{\mathrm{AD}}=0.7 \mathrm{~nm}$, $V_{\mathrm{AD}}=0.04 \mathrm{eV}(3), r_{\mathrm{AD}}=1.0 \mathrm{~nm}, V_{\mathrm{AD}}=0.008 \mathrm{eV}$ (4). Parabolic terms of the locally excited and charge transfer states $\lambda s^{2}$ and $\lambda(1-s)^{2}$ were used. (Bottom) Dependences of the reorganization energy, $\lambda$ (1), electronic coupling, $V_{\mathrm{AD}}$ (2), and intermolecular Lennard-Jones potential, $U_{\mathrm{LJ}}(3)$, on the distance between reactant molecules, $r_{\mathrm{AD}}$ (see the Appendix).

The goal of this paper is to demonstrate that many kinds of excited-state ET reactions actually follow the transient exciplex (contact) mechanism rather than onestep long-range mechanism. In these cases adiabatic ET occurs, strong electronic coupling promotes medium and reactants reorganization consistent with gradual charge shift, and single-step Marcus and radiationless transition theories fail. This mechanism causes some specific features of ET kinetics, which can be used as criteria to distinguish this mechanism from other mechanisms of ET, and explains many experimental peculiarities, discussed earlier as "puzzles of electron transfer" [30].

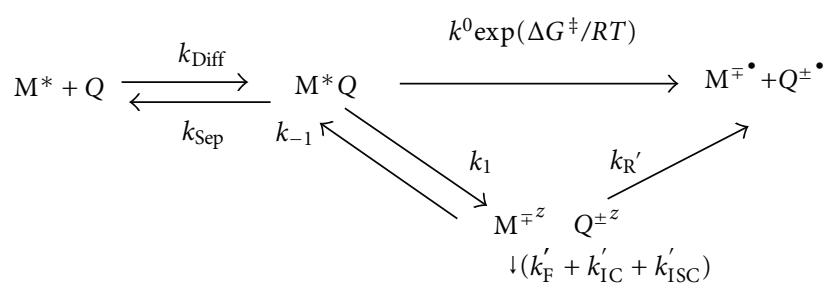

SCHEMe 1

\section{Kinetics and Energetics of Exciplex Formation and Dissociation into Radical Ions}

Conventional approaches [31] describe ET reactions as consecutive steps of diffusion-controlled formation of loose re-actants pair and ET in this pair (the top line of Scheme 1).

In such a case ET rate constant (or quenching constant, $k_{\mathrm{Q}}$, usually measured for excited-state reactions) can be expressed as

$$
k_{\mathrm{Q}}=\frac{1}{\left[1 / k_{\text {Diff }}+\left(1 / K_{\text {Ass }} k^{0}\right) \exp \left(\Delta G^{\ddagger} / R T\right)\right]},
$$

where $K_{\mathrm{Ass}}=k_{\text {Diff }} / k_{\text {Sep }}$ and $k_{\text {Diff }}$ and $k_{\text {Sep }}$ are diffusion association and separation rate constants, $k^{0}$ is preexponential factor, and $\Delta G^{\ddagger}$ is activation energy of ET step. According to the Marcus approach $\Delta G^{\ddagger}$ can be represented as a parabolic function of the free energy of $\mathrm{ET}\left(\Delta G_{\mathrm{ET}}\right)$ and reorganization energy $\lambda[5]$

$$
\begin{gathered}
\Delta G^{\ddagger}=\frac{\left(\lambda+\Delta G_{\mathrm{ET}}\right)^{2}}{4 \lambda}, \\
k_{\mathrm{Q}}=\frac{1}{\left\{1 / k_{\text {Diff }}+\left(1 / K_{\mathrm{Ass}} k^{0}\right) \exp \left[\left(\lambda+\Delta G_{\mathrm{ET}}\right)^{2} / 4 \lambda R T\right]\right\}} .
\end{gathered}
$$

Here total reorganization energy $\lambda=\lambda_{\mathrm{S}}+\lambda_{\mathrm{i}}$, internal reorganization energy of reactants $\lambda i=1 / 2 \sum_{j} h v_{j} \Delta q_{j}{ }^{2}$, (where $\nu_{j}$ and $\Delta q_{j}$ are frequencies of the vibrational modes and differences of their dimensionless coordinates minima between LE and CT states), and medium reorganization energy

$$
\lambda_{S}=1.44\left(\frac{1}{2 \rho_{\mathrm{M}}}+\frac{1}{2 \rho_{\mathrm{Q}}}-\frac{1}{r_{\mathrm{MQ}}}\right)\left(\frac{1}{n^{2}}-\frac{1}{\varepsilon}\right),
$$

where $\rho_{\mathrm{M}}$ and $\rho_{\mathrm{Q}}$ are radii of reactant molecules, $r_{\mathrm{MQ}}$ is a distance between them, $n$ and $\varepsilon$ are refractive index and dielectric permeability of the medium, respectively. This approach implies that $k_{\mathrm{Q}}$ should decrease in both cases: when free energy of electron transfer $\Delta G_{\mathrm{ET}} \gg-\lambda$ and when $\Delta G_{\mathrm{ET}} \ll-\lambda$ (the so called "Marcus inverted region").

Experimental data of Rehm and Weller [31] demonstrated some different (hyperbolic) dependence $\Delta G^{\ddagger}$ on $\Delta G_{\mathrm{ET}}$

$$
\Delta G^{\ddagger}=\frac{\Delta G_{\mathrm{ET}}}{2}+\left[\left(\frac{\Delta G_{\mathrm{ET}}}{2}\right)^{2}+\left(\Delta G_{0}^{\ddagger}\right)^{2}\right]^{1 / 2},
$$




$$
k_{\mathrm{Q}}=\frac{1}{\left\{\left(1 / k_{\mathrm{Diff}}\right)+\left(1 / K_{\mathrm{Ass}} k^{0}\right) \exp \left[\left(\Delta G_{\mathrm{ET}} / 2+\left(\left(\Delta G_{\mathrm{ET}} / 2\right)^{2}+\left(\Delta G_{0}^{\ddagger}\right)^{2}\right)^{1 / 2}\right) / R T\right]\right\}},
$$

where $\Delta G_{0}^{\ddagger}$ is an activation energy for isoergonic reaction. This dependence was proposed to explain the absence of the decrease of experimental $k_{\mathrm{Q}}$ at strongly negative $\Delta G_{\mathrm{ET}}$ (the absence of the "Marcus inverted region"). Experimental value $\Delta G_{0}^{\ddagger} \approx 0.11 \mathrm{eV}$ was found for large set of donoracceptor systems in acetonitrile solution $\left(\varepsilon=37, n^{2}=1.8\right)$ [31].

Radiationless transition mechanism [7-9] does not require any preliminary activation and occurs, when $\Delta E=$ $-\left(\Delta G_{\mathrm{ET}}{ }^{*}+\lambda\right)>0$. Its rate depends on the frequencies $\nu_{V}$ and reorganization energies $\lambda_{V}$ of vibrational modes involved into the transition. Usually one of these modes can be considered as dominating and rate constant can be expressed as

$$
\begin{aligned}
k_{\mathrm{ET}}= & \left(\frac{4 \pi^{2}}{h}\right) V_{\mathrm{AD}}^{2}\left[\frac{1}{\sigma(2 \pi)^{1 / 2}}\right] \\
& \times \sum_{m}\left\{\left[\frac{\exp (-S) \times S^{m}}{m !}\right] \times \exp \left[\frac{-\left(\Delta E_{\mathrm{ET}}-m h v_{V}\right)^{2}}{2 \sigma^{2}}\right]\right\},
\end{aligned}
$$

where $V_{\mathrm{AD}}$ is an electronic coupling matrix element, responsible for the transition, $S=\lambda_{V} / h \nu_{V}=(\Delta q)^{2} / 2, q$ and $\Delta q$ are the dimensionless coordinate, corresponding to this high-frequency mode, and the difference of the positions of the minima along this coordinate for potential curves for LE and CT electronic states $\left(U_{\mathrm{LE}}=h \nu_{\mathrm{VLE}} q^{2}, U_{\mathrm{CT}}=h \nu_{\mathrm{VCT}}\right.$ $\left.(q-\Delta q)^{2}\right)$, respectively, $\lambda_{V}$ is the reorganization energy for dominating high-frequency $\left(\nu_{V}\right)$ vibrational mode, $\sigma$ is spectroscopic width of the vibrational level frequently assumed as $\sigma^{2}=2 \lambda_{S} k_{\mathrm{B}} T, \lambda_{S}$ is the medium reorganization energy, $h$ and $k_{\mathrm{B}}$ are Plank and Boltzmann constants, $T$ is temperature. Probably this mechanism dominates at $\Delta G_{\mathrm{ET}}<$ $-\lambda$ for ET between uncharged molecules [32].

Transient exciplex $\left(\mathrm{M}^{\mp z} \mathrm{Q}^{ \pm z}\right)$ formation mechanism (bottom line of Scheme 1) was discussed by several groups of authors [10-17] to explain some experimentally observed deviations from Marcus and Weller relationships between $k_{\mathrm{Q}}$ and $\Delta G_{\mathrm{ET}}$. Here $k_{\mathrm{F}}^{\prime}, k_{\mathrm{IC}}^{\prime}, k_{\mathrm{ISC}}^{\prime}$, and $k_{\mathrm{R}}^{\prime}$ are rate constants of fluorescence, internal conversion, intersystem crossing, and dissociation of the exciplex into radical ions, respectively; $z$ is a degree of charge transfer. Figure 1 demonstrates that in contact pairs of reactant molecules $\left(r_{\mathrm{AD}}<0.4 \mathrm{~nm}\right)$ at strong coupling (electronic coupling matrix element $V_{\mathrm{AD}}>0.2 \mathrm{eV}$ ) the energy minimum corresponding to exciplex formation $\left(\Delta G_{\mathrm{Ex}}{ }^{*}\right)$ appears instead of the maximum, corresponding to the transition state in the long-range ET mechanisms.

An important feature of the transient exciplex mechanism of the excited-state ET is a large contribution of radiationless (internal conversion and intersystem crossing) and radiative processes, which compete with exciplex dissociation into radical ions

$$
\tau_{0}^{\prime}=\frac{1}{\left(k_{\mathrm{R}}^{\prime}+k_{\mathrm{IC}}^{\prime}+k_{\mathrm{ISC}}^{\prime}+k_{\mathrm{F}}^{\prime}\right)} .
$$

For this reason

$$
\Delta G_{\mathrm{Ex}}^{\ddagger} \approx 2.3 R T\left(\log \left(\frac{k_{\mathrm{B}} T}{h}\right)+\log \left(k_{\mathrm{R}}^{\prime}+k_{\mathrm{IC}}^{\prime}+k_{\mathrm{ISC}}^{\prime}+k_{\mathrm{F}}^{\prime}\right)\right) .
$$

These decay processes become very important (see Section 3) since $1 / k_{\mathrm{R}}^{\prime}$ can reach $1-100 \mathrm{~ns}$ (in contrast to ultrashort lifetime of the transition state).

Complete treatment of this adiabatic reaction dynamics requires sophisticated consideration of simultaneous medium reorganization and gradual charge shift, taking into account a feedback between them, and cannot be done in terms of the diabatic approaches. Fortunately, a steady-state approach provides the possibility to describe total reaction rate in terms of only two parameters: free energy $\Delta G_{\mathrm{Ex}}{ }^{*}$ of the transient exciplex formation from $\mathrm{M}^{*}$ and $\mathrm{Q}$ and its lifetime $\tau_{0}^{\prime}$

$$
k_{\mathrm{Q}}=\frac{1}{\left[1 / k_{1}+\tau_{0}^{\prime} \exp \left(\Delta G_{\mathrm{Ex}}{ }^{*} / R T\right)\right]},
$$

where $k_{1}(\leq k$ Diff $)$ is a rate constant of the exciplex formation. This approximation is valid when $k_{\mathrm{Q}}<$ $\left(k_{\text {Diff }} \exp \left(-\Delta G_{\mathrm{Ex}}{ }^{*} / R T\right)+1 / \tau_{0}^{\prime}\right)$. Both $\Delta G_{\mathrm{Ex}}{ }^{*}$ and a degree of charge transfer in the exciplex, $z$, depend on $\Delta G_{\mathrm{ET}}{ }^{*}$ and medium polarity [34-41]. This approach was developed in $[10,11,15,16]$ by simultaneous study of kinetics of an excited state quenching and an exciplex formation and decay. Some earlier approach to the transient exciplex formation [42] considered constant $z$ in the exciplex independent of $\Delta G_{\mathrm{ET}}$ which provided erroneous dependence of $\Delta G_{\mathrm{Ex}}{ }^{*}$ on $\Delta G_{\mathrm{ET}}{ }^{*}$. Another approach [43] considered some average values of $\Delta G_{\mathrm{Ex}}{ }^{*}$ and $\tau_{0}^{\prime}$ along the reaction coordinate, that implied very shallow potential minimum for an exciplex and also provided erroneous dependence of $\Delta G_{\mathrm{Ex}}{ }^{*}$ on $\Delta G_{\mathrm{ET}}{ }^{*}$. Experimental width of exciplex emission spectra [44, 45], close to that for ordinary aromatic compounds, and wellknown exponential kinetics of their decay indicate that exciplexes represent ordinary chemical species with distinct energy minimum, and kinetics of their formation and decay can be treated in terms of ordinary chemical kinetics and requires no complications related to the averaging which could be necessary for very flat potential surface. Model plots of $\Delta G$ along the reaction coordinate are shown in Figure 2. They are drawn for isoergonic $\left(\Delta G_{\mathrm{ET}}{ }^{*}=0\right)$ excited-state ET and demonstrate the difference between transient exciplex 
$\left(\Delta G_{\mathrm{Ex}}{ }^{*}=-0.15 \mathrm{eV}, V_{\mathrm{AD}}=0.2 \mathrm{eV}\right)$ and long-range $\left(\Delta G^{\ddagger}=\right.$ $0.45 \mathrm{eV}, \lambda=1.8 \mathrm{eV}$ ) mechanisms (standard expression for diffusion barriers was used-see the Appendix).

Thermodynamic parameters $(\Delta G, \Delta H$, and $\Delta S)$ of exciplex formation and its conversion into the reaction products were studied experimentally by investigation of temperature dependences of fluorescence quantum yields and decay kinetics of parent excited molecules $\mathrm{M}^{*}$ and exciplex $[15,45-47]$. The dependences of $\ln \left\{\left(\varphi_{0} / \varphi-1\right) / \tau_{0}[Q]\right\}$ and $\ln \left\{\left(\varphi^{\prime} / \varphi\right) /[Q]\right\}$ versus $1 / T$ (where $\varphi_{0}$ and $\varphi$ are the fluorescence quantum yields of $\mathrm{M}^{*}$ in the absence and in the presence of the quencher $\mathrm{Q}, \varphi^{\prime}$ is the fluorescence quantum yield of exciplex, and $\tau_{0}$ is the fluorescence lifetime of $\mathrm{M}^{*}$ in the absence of Q) have a bell shape (Figure 3) [15, 45-47]:

$$
\begin{aligned}
\ln \left\{\frac{\left(\varphi_{0} / \varphi-1\right)}{\tau_{0}[\mathrm{Q}]}\right\}= & \ln k_{1}-\ln \left(1+k_{-1} \tau_{0}^{\prime}\right) \\
= & A+\frac{C}{T}-\ln \left[1+B \exp \left(\frac{D}{T}\right)\right] \\
= & \ln {k_{1}}^{0}+\frac{\Delta S_{1}^{\ddagger}}{R}-\frac{\Delta H_{1}^{\ddagger}}{R T} \\
& -\ln \left\{1+\exp \left[\frac{\left(\Delta S_{-1}^{\ddagger}-\Delta S_{\mathrm{Ex}}^{\ddagger}\right)}{R}\right.\right. \\
& \left.\left.-\frac{\left(\Delta H_{-1}^{\ddagger}-\Delta H_{\mathrm{Ex}}^{\ddagger}\right)}{R T}\right]\right\},
\end{aligned}
$$

$$
\begin{aligned}
\ln \left\{\frac{\left(\varphi^{\prime} / \varphi\right)}{[\mathrm{Q}]}\right\}= & \ln \left(\frac{k_{\mathrm{F}}^{\prime}}{k_{\mathrm{F}}}\right)+\ln \left(\frac{k_{1}}{k_{-1}}\right)-\ln \left(1+\frac{1}{k_{-1} \tau_{0}^{\prime}}\right) \\
= & A^{\prime}+\frac{C^{\prime}}{T}-\ln \left[1+B^{\prime} \exp \left(\frac{D^{\prime}}{T}\right)\right] \\
= & \ln \left(\frac{k_{\mathrm{F}}^{\prime}}{k_{\mathrm{F}}}\right)+\frac{\Delta S_{\mathrm{Ex}}^{*}}{R}-\frac{\Delta H_{\mathrm{Ex}}^{*}}{R T} \\
& -\ln \left\{1+\exp \left[\frac{\left(\Delta S_{\mathrm{Ex}}^{\ddagger}-\Delta S_{-1}^{\ddagger}\right)}{R}\right.\right. \\
& \left.\left.+\frac{\left(\Delta H_{-1}^{\ddagger}-\Delta H_{\mathrm{Ex}}^{\ddagger}\right)}{R T}\right]\right\} .
\end{aligned}
$$

Here $\Delta S_{1}^{\ddagger}, \Delta H_{1}^{\ddagger}, \Delta S_{-1}^{\ddagger}, \Delta H_{-1}^{\ddagger}, \Delta S_{\mathrm{Ex}}^{\ddagger}, \Delta H_{\mathrm{Ex}}^{\ddagger}$ are entropies and enthalpies of activation of exciplex formation, dissociation into parent reactants, and decay; $\tau_{0}^{\prime}$ is the exciplex lifetime; $k_{1}{ }^{0}=k_{\mathrm{B}} T / h$ is preexponential factor; $k_{\mathrm{F}}$ and $k_{\mathrm{F}}^{\prime}$ are emission rate constants for parent excited molecules and exciplex. Low-temperature wing of this dependence has a negative slope, which corresponds to $\Delta H_{1}^{\ddagger}$ of the diffusion-controlled exciplex formation reaction (activation enthalpy of the exciplex formation $\Delta H^{\ddagger}>0$ ) and hightemperature wing has a positive slope, which corresponds to

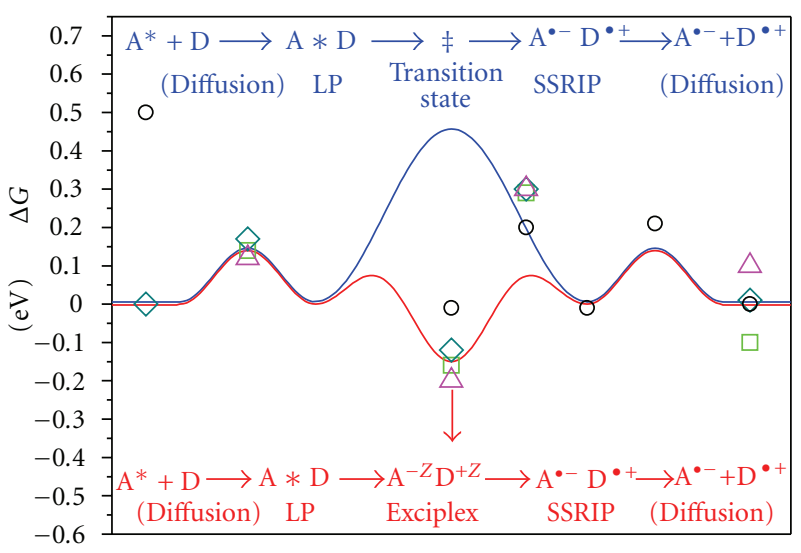

FIGURE 2: Schematic plot of free energy along the reaction pathway $\left(\mathrm{A}^{*}+\mathrm{D} \rightleftarrows \mathrm{A}^{*} \cdots \mathrm{D}\right.$ (loose pair of reactants) $\rightleftarrows \mathrm{A}^{-z} \mathrm{D}^{+z}$ (transition state or exciplex) $\rightleftarrows \mathrm{A}^{\bullet-} \ldots \mathrm{D}^{\bullet+}$ (solvent separated ion pair) $\rightleftarrows \mathrm{A}^{\bullet-}+\mathrm{D}^{\bullet+}$ ) blue line-for long-range mechanism ((2), $\left.\lambda=2.0 \mathrm{eV}, \Delta G_{\mathrm{ET}}^{\ddagger}=0.5 \mathrm{eV}\right)$; and red line-for transient exciplex mechanism $\left(\Delta G_{\mathrm{Ex}}{ }^{*}=-0.15 \mathrm{eV}\right)$ for isoenergetic ET reaction $\left(\Delta G_{\mathrm{ET}}{ }^{*}=0\right)$ in butyronitrile $\left(\Delta G_{\mathrm{Diff}}^{\ddagger}=0.14 \mathrm{eV}\right)$. Experimental data on $\Delta G_{1}^{\ddagger}, \Delta G_{\mathrm{Ex}}{ }^{*}, \Delta G_{\mathrm{Ex}}^{\ddagger}$ along the reaction coordinate: green squares-for 9-cyanoanthracene* $+1,8$-dimethylnaphthalene in $\operatorname{MeCN}\left(\Delta G_{\mathrm{ET}}{ }^{*}=-0.1 \mathrm{eV}\right)$; dark cyan rhombs-in PrCN $\left(\Delta G_{\mathrm{ET}}{ }^{*}=0.01 \mathrm{eV}\right)$; magenta triangles-for 9-cyanophenanthrene +1 , 2-dimethoxylbenzene in $\operatorname{PrCN}\left(\Delta G_{\mathrm{ET}}{ }^{*}=0.1 \mathrm{eV}\right)$ [15]; black circles-for 1, 2, 4, 5-tetracyanobenzene* + durene in 1, 2-dichloroetane (according to the experimental data [27, 28], $\left.\Delta G_{\mathrm{ET}}^{*} \approx-0.5 \mathrm{eV}\right)$.

$\Delta H_{\text {Ex }}{ }^{*}=\Delta H_{1}^{\ddagger} / \Delta H_{-1}^{\ddagger}<0$ and equilibrium between transient exciplex and parent reactants (when $T\left(\Delta S_{-1}^{\ddagger}-\Delta S_{\mathrm{Ex}}^{\ddagger}\right)>$ $\left.\left(\Delta H_{-1}^{\ddagger}-\Delta H_{\mathrm{Ex}}^{\ddagger}\right)\right)$. Experimental investigations of the kinetics of exciplex formation and decay demonstrate that a decrease of the exciplex concentration and emission quantum yield with the rise of temperature is caused by the increase of the rate of its dissociation into parent reactants, rather than by the decrease of its formation rate or increase of its decay rate $[15,29,32,37,38,45-47]$. In such a case the quenching rate is controlled by thermodynamics of the exciplex formation and decay rather than by Marcus reorganization barrier.

Experimental dependence of $\Delta G_{\mathrm{Ex}}{ }^{*}$ on $\Delta G_{\mathrm{ET}}{ }^{*}$ and medium polarity can be described by a model of selfconsistent polarization of the medium and reactants $[34,37]$, that takes into account a feedback between a degree of charge transfer in the exciplex and a degree of medium reorganization. According to this model free energy of exciplex formation and free energy of electron transfer can be expressed as functions of the degree of charge transfer $z$ in the exciplex

$$
\begin{aligned}
\Delta G_{\mathrm{Ex}}^{*}= & U_{\mathrm{Ex}}-U_{\mathrm{LE}}{ }^{0}=z_{\mathrm{M}}^{2}\left(\frac{\mu_{0}^{2}}{\rho^{3}}\right) \mathrm{f}(\varepsilon) \\
& -\frac{V_{\mathrm{AD}}}{\left(1 / z_{\mathrm{Ex}}-1\right)^{1 / 2}}+a^{*},
\end{aligned}
$$




$$
\begin{aligned}
\Delta G_{\mathrm{ET}}{ }^{*}= & V_{\mathrm{AD}}\left[\left(\frac{1}{z}-1\right)^{1 / 2}-\frac{1}{(1 / z-1)^{1 / 2}}\right] \\
& +(2 z-1)\left(\frac{\mu_{0}^{2}}{\rho^{3}}\right) \mathrm{f}(\varepsilon) .
\end{aligned}
$$

Here $\mathrm{f}(\varepsilon)=(\varepsilon-1) /(\varepsilon+2)$, when $\varepsilon$ is dielectric permittivity of the medium, $\mu_{0}$ is a dipole moment of CT state, $\rho$ is a radius of the exciplex solvent shell, $V_{12}$ is electronic coupling, and $a^{*}$ is intermolecular repulsion in the exciplex (including the entropy term of contact pair formation). This pair of equations provides in implicit form the relationship between $\Delta G_{\mathrm{Ex}}{ }^{*}$ and $\Delta G_{\mathrm{ET}}{ }^{*}$, which differs essentially from a simple model of electronic coupling of LE and CT states, independent of the degree of the solvent polarization. More

sharp increase of $z$ and spectral shift of the emission spectra of the exciplex, relative to the parent LE excited state $h \Delta v=h\left(v_{\max }-v_{\max }^{\prime}\right)$, is observed in more narrow range of $\Delta G_{\mathrm{ET}}{ }^{*}$ [34-38], due to feedback between the degree of charge transfer in the exciplex and degree of the medium polarization. Nevertheless this dependence can be approximated (Figure 4) by explicit hyperbolic function, which are more convenient for practical use than implicit form $[16,37]$

$$
\Delta G_{\mathrm{Ex}} * \approx a+\frac{\left(\Delta G_{\mathrm{ET}}^{*}+b\right)}{2}-\left[\frac{\left(\Delta G_{\mathrm{ET}}^{*}+b\right)^{2}}{4}+c^{2}\right]^{1 / 2}
$$

$$
k_{\mathrm{Q}}=\frac{1}{\left.\left\{1 / k_{1}+\exp \left[\ln \tau_{0}^{\prime}+\left(a+\left(\Delta G_{\mathrm{ET}}{ }^{*}+b\right) / 2-\left(\left(\Delta G_{\mathrm{ET}}{ }^{*}+b\right)^{2} / 4+c^{2}\right)^{1 / 2}\right) / R T\right)\right]\right\}} .
$$

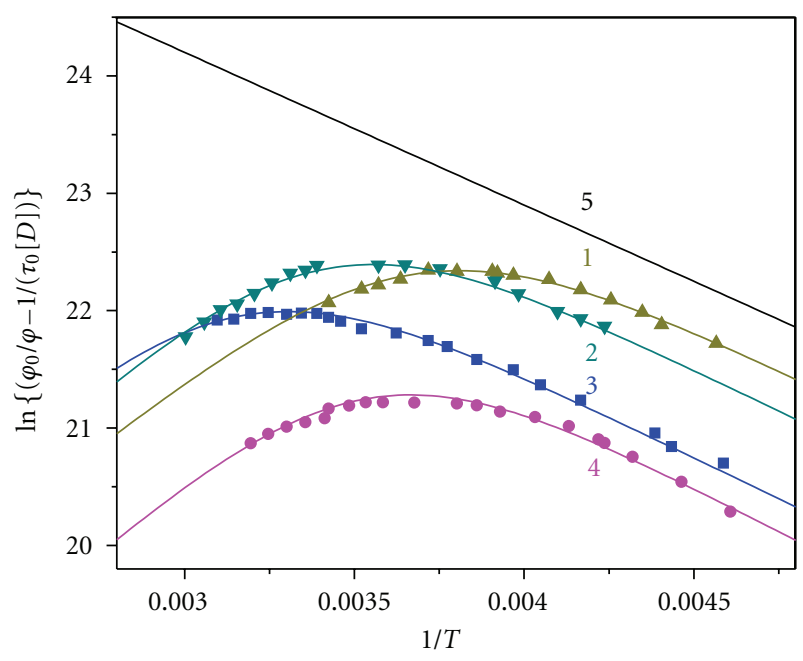

Figure 3: Temperature dependence of $\ln K_{S V}=\ln \left(\left(\varphi_{0} / \varphi-\right.\right.$ $1) /[\mathrm{Q}])$ for quenching of 9-cyanoanthracene (1-3) and 1, 12benzperylene (4) fluorescence by 1, 6-dimethylnaphthalene (1), 1, 4-dimethoxybenzene (2), 1, 3, 5-trimethoxybenzene (3), and 1, 2, 4-trimethoxybenzene (4) in butyronitrile [15]. The line labelled 5 shows the temperature dependence of the diffusion rate constant.

Here parameter $a$ can be attributed approximately to the sum of intermolecular repulsion and entropy of the contact reactant pair formation. Parameter $b$ reflects the medium polarity effect and corresponds to the difference of free energies of a dipole solvation $\left(\left(\mu_{0}^{2} / \rho^{3}\right) \mathrm{f}(\varepsilon)\right)$ between the given solvent and a standard solvent for the determination of $\Delta G_{\mathrm{ET}}{ }^{*}$. Parameter $c$ occurs to be some smaller than $V_{12}$. Experimental values of $\Delta G_{\mathrm{Ex}}{ }^{*}$ and $h \Delta v$ were found to be described well by this model [34-38].

Obtained values of $\Delta G_{1}^{\ddagger}, \Delta G_{\mathrm{Ex}}{ }^{*}$, and $\Delta G_{\mathrm{Ex}}^{\neq}$provide a possibility to compare experimental data and model

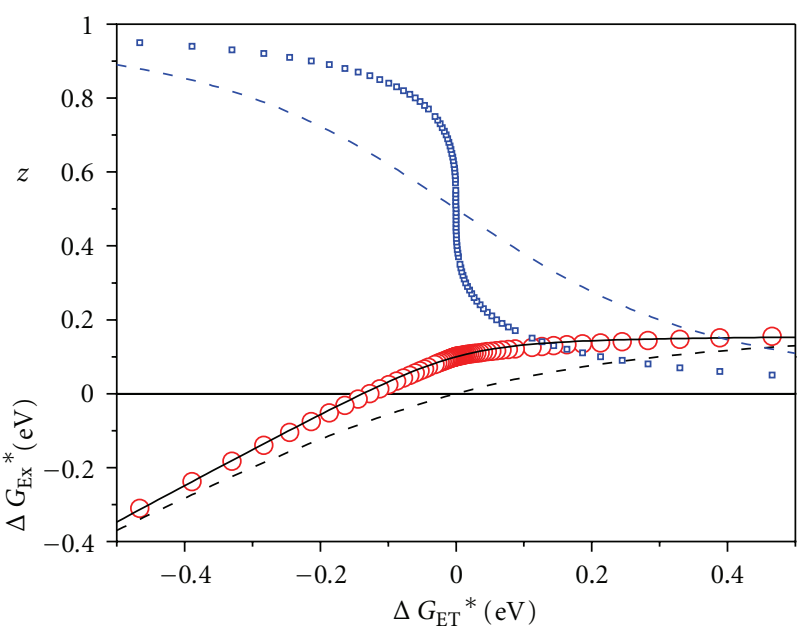

FIGURE 4: Simulation of the dependences $\Delta G_{\mathrm{Ex}}{ }^{*}$ (red points) and $z$ (blue points) versus $\Delta G_{\mathrm{ET}}{ }^{*}$, according to self-consistent polarization of the medium and exciplex ((13), (14), $V=0.2 \mathrm{eV}, m f(\varepsilon)=$ $0.4 \mathrm{eV})$. Approximation of $\Delta G_{\mathrm{Ex}}{ }^{*}-\Delta G_{\mathrm{ET}}{ }^{*}$ dependence (black line) by hyperbolic function (15). Dash lines show dependences of $\Delta G_{\mathrm{Ex}}{ }^{*}$ and $z$ from $\Delta G_{\mathrm{ET}}{ }^{*}$ according to the simple model of interaction of $\operatorname{LE}\left(U_{\mathrm{LE}}{ }^{0}=0+a^{*}\right)$ and $\mathrm{CT}\left(U_{\mathrm{CT}}^{0}=\Delta G_{\mathrm{ET}}{ }^{*}+a^{*}\right)$ terms.

profiles, shown in Figure 2. Diffusion barriers for the exciplex formation $\left(\Delta G_{1}^{\ddagger}\right)$ were found to be close to the ordinary diffusion values $(0.15 \mathrm{eV})$. But activation barrier for exciplex dissociation into radical ions occurs to be some greater $(0.2-0.3 \mathrm{eV})$ and exceeds that estimated from the rate of dissociation of ion pair by continual dielectric model (see the Appendix). Obviously, this model underestimates the barrier related to the solvent shell reorganization during the conversion of the contact radical ion pair into the solvent-separated pair. Values of $\Delta G_{\mathrm{Ex}}{ }^{*}$ are in the range $-(0.12-0.2) \mathrm{eV}$ since for these systems electronic coupling 


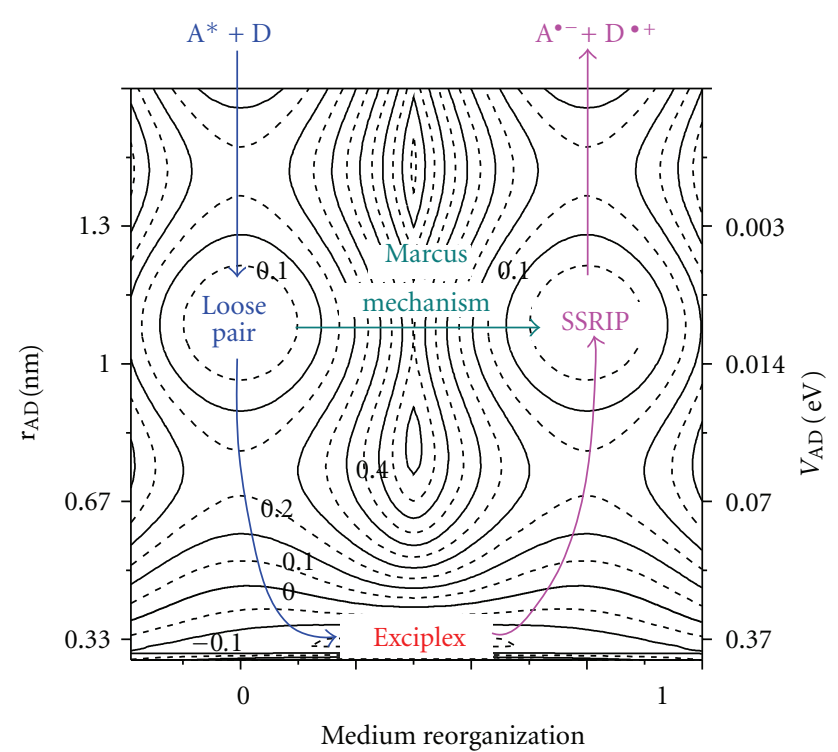

Figure 5: Schematic map of the dependence of the free energy on the distance between reactant molecules and medium reorganization for isoergonic ET reaction (see the Appendix).

provides considerable contribution into $\Delta G_{\mathrm{Ex}} *$ because of small energy gap $\Delta G_{\mathrm{ET}}{ }^{*}=0 \pm 0.1<V_{\mathrm{AD}} \approx 0.2 \mathrm{eV}$. This figure presents also experimental values for activation barriers of exciplex conversion into solvent-separated radical ion pairs (SSRIP) $\left(\Delta G_{\text {CRIP } \rightarrow \text { SSRIP }}^{\ddagger}\right)$ and its dissociation into free radical ions $\left(\Delta G_{\text {SSRIP } \rightarrow \text { FRI }}^{\ddagger}\right)$ for more exergonic $\left(\Delta G_{\mathrm{ET}}{ }^{*} \approx-0.5 \mathrm{eV}\right)$ ET reaction of 1, 2, 4, 5-tetracyanobenzene and various methylbenzenes. These data were obtained by Gould et al. [28] by investigation of kinetics of exciplex emission and kinetics of absorption of exciplex, ion pair, and free radical ions in various solvents at room temperature. In this case the energy gap between LE and CT states exceeds $0.5 \mathrm{eV}$, and energies of the exciplex (CRIP) and SSRIP are close to each other. It was found that the rates of recombination in both kinds of radical ion pairs were comparable or even greater than the rate of dissociation of radical ion pairs. This indicates that charge recombination in CIP (internal conversion in exciplexes) and SSRIP is the main route of the excited-state quenching.

According to the obtained experimental data we can draw a model three-dimensional surface of $\Delta G$ versus $r_{\mathrm{AD}}$ and medium reorganization coordinates for ET reaction, which includes transient exciplex as well as long range mechanisms of ET, using the same parameters as in Figure 1. Figures 5 and 6 present a map and general view of such surface for isoergonic reaction. One can see that for such reaction transient exciplex (contact) mechanism has significantly smaller activation energy relative to preliminary reorganization (long range) mechanism.

\section{Transient Exciplex Decay}

Significance of the radiationless decay processes for photochemistry and photophysics of exciplexes was recognized immediately as exciplexes were discovered [23-26]. It is well known that only few donor-acceptor pairs can form exciplexes, having specific emission band. For instance, excited aromatic acceptors form fluorescent exciplexes only with tertiary amines, while primary and secondary amines quench their fluorescence, but exhibit no exciplex fluorescence. This phenomenon was attributed to both very short lifetime of exciplexes of primary and secondary amines [24-26] and a decrease of the yield of their formation because of the competing quenching [23], promoted by weak $\mathrm{N}-\mathrm{H}$ bonds in cation radicals of primary and secondary amines. For this reason no photoreactions were observed in such systems.

A key stage of radical ions formation is the dissociation of an exciplex (and SSRIP). Rates of this dissociation were studied comprehensively by Gould et al. [27, 28] for the reactions of excited tetracyanobenzene with various methylbenzenes $\left(\Delta G_{\mathrm{ET}}{ }^{*}=-(0.5-0.9) \mathrm{eV}\right)$ in various solvents $(\varepsilon=7-25)$. It was found that activation energy of transformation of CIP into SSRIP linearly depended on $\Delta G_{\text {CIP/SSRIP }}$ and solvent polarity and practically did not depend on $\Delta G_{\mathrm{ET}}{ }^{*}$ in this range of $\Delta G_{\mathrm{ET}}{ }^{*}: \Delta G_{\mathrm{CIP} \rightarrow \mathrm{SSRIP}}^{\neq} \approx$ $0.22+0.75 \Delta G_{\mathrm{CIP} \rightarrow \mathrm{SSRIP}}, \Delta G_{\mathrm{CIP} \rightarrow \mathrm{SSRIP}}^{\ddagger} \approx 0.16+0.72 / \varepsilon \approx$ $0.48-0.66(\varepsilon-1) / 2(\varepsilon+2) \approx 0.68-1.05(\varepsilon-1) /(2 \varepsilon+1))$. These data indicate the possibility to treat the separation of radical ions in terms of common diffusion kinetics with activation barrier $(0.19-0.26 \mathrm{eV})$, which depends on the solvent polarity (in the range $\varepsilon=7-25$ ) rather than on $\Delta G_{\mathrm{ET}}{ }^{*}$. Solvent polarity dependence of $\Delta G_{\mathrm{CIP} \rightarrow \text { SSRIP }}^{\ddagger}$ corresponds to rather small increase of intermolecular distance for transition state, relative to CIP (ca. $0.1 \mathrm{~nm}$ ).

Ultrafast decay of transient exciplexes, containing hydrogen bond, was studied by ps and fs spectroscopy and discussed by Mataga et al. [50-52] for 1-aminopyrene-pyridine and dibenzocarbazole-pyridine. They found that hydrogen bonding facilitated both ET (yielding radical ion pair) and charge recombination in these pairs. Probably strong electronic coupling in hydrogen-bonded complexes increases both charge separation and recombination rates (due to the radiationless decay, promoted by weak $\mathrm{N}-\mathrm{H}$ bond in pyridinium cation radical fragment). The total result of both these effects was a very strong acceleration of the quenching rate relative to similar systems without hydrogen bond.

The problem of radiationless transitions, competing with the formation of exciplexes or controlling their lifetimes, is a part of more general problem of nonadiabatic transitions between potential surfaces in photochemistry and photophysics. Occurrence of intersections between molecular potential energy surfaces was recognized already in the middle of the 20th century [53-55]. Importance of conical intersections of potential surfaces of excited and ground states for the quenching of excited states was discussed in the middle sixties. Nikitin and Bjerre $[54,55]$ showed that efficient quenching of excited sodium atoms $\mathrm{Na}^{*}\left({ }^{2} \mathrm{P}\right)$ by molecular nitrogen $\mathrm{N}_{2}$ occurred due to crossing of ionic term $\mathrm{Na}^{+} \mathrm{N}_{2}^{-}\left({ }^{3} \sum_{+} u^{+}\right)$with both the excited-state term $\mathrm{Na}^{*}\left({ }^{2} P\right) \mathrm{N}_{2}\left({ }^{1} \sum \mathrm{g}^{+}\right)$and the ground-state term $\mathrm{Na}\left({ }^{2} S\right)$ $\mathrm{N}_{2}\left({ }^{1} \sum \mathrm{g}^{+}\right)$. Key role of these intersections ("funnels") for organic photochemistry was discussed by Michl [56-61] and 




Figure 6: Schematic tridimensional diagram of the dependence of the free energy on the distance between reactant molecules and medium reorganization for isoergonic ET reaction (see the Appendix).

other authors (see, for instance, $[62,63]$ ). Similar hypothesis has been proposed [23] to explain why primary and secondary amines quench efficiently the fluorescence of many aromatic compounds without the appearance of the emission of exciplexes and the formation of radical ions, unlike the tertiary amines, which yield fluorescing exciplexes. In the case of exciplexes such intersections arise, when some bond in the radical ion of a donor or an acceptor has much smaller dissociation energy, relative to the parent ground state. For instance, ionogenic $\mathrm{N}-\mathrm{H}$ bond in cation radicals of primary and secondary amines easily dissociates in contrast to $\mathrm{N}-\mathrm{C}$ bond in cation radicals of tertiary amines. Such phenomena reduce drastically the quantum yield of the formation of exciplexes and/or their lifetime [23-28] and increase the quenching rate constant (for instance, see below the discussion of quenching of the excited cyanoaromatics by pyridine).

Formal kinetics does not require special term for an induced internal conversion $\left(k_{\mathrm{i}}\right)$ to describe an excited state quenching, since this term can be involved formally into the total quenching constant together with rate constant $k_{\mathrm{Q}}$. But the rate constants $k_{\mathrm{i}}$ and $k_{\mathrm{Q}}$ have a different physical behaviour and a different temperature dependence, which can be used for their distinction. Marcus mechanism of ET requires positive activation enthalpy, related to the preliminary medium reorganization, and $k_{\mathrm{Q}}$ rises with temperature. In contrast, induced internal conversion requires no activation enthalpy, and $k_{\mathrm{i}}$ should not depend on temperature (similarly to the radiationless transition mechanism). Quantum yields of radical ion pair are also different in these cases. For long-range mechanism the yield should be close to 1 (although final quantum yield of radical ions can be some smaller because of their recombination in the pair). But induced internal conversion, yielding directly a ground state of reactants, can reduce this yield significantly.

Even in the absence of real intersections the decrease of some bond energy in CT state (exciplex) and/or the increase of equilibrium distance of this bond, relative to the ground state, will promote internal conversion because of strong increase of the overlap integral of vibrational wave function of CT and ground states. This causes a strong increase of the transition rate and decreases the exciplex lifetime, when any bond in an exciplex has substantially smaller dissociation energy or substantially larger equilibrium distance, relative to the ground-state pair of reactant molecules. The increase of both the internal conversion and the intersystem crossing rates was observed even for fluorescent exciplexes, relative to similar characteristics in the parent excited molecules $[28,45]$.

Actually the decrease of exciplex lifetime and exciplex yield has similar physical behavior related to the weakening of some bonds in CT state and conical intersections of LE, $\mathrm{CT}$, and ground states. The decrease of an exciplex yield becomes more important for exergonic ET reactions, when the energy gap between LE and CT states is large enough. But in these cases $k_{\mathrm{Q}}$ for bimolecular reactions is close to the diffusion limit, and a decrease of the exciplex yield affects quantum yields of photoproducts formation, but has very weak influence on the value of $k_{\mathrm{Q}}$. In contrast, a decrease of exciplex lifetime significantly affects $k_{\mathrm{Q}}$ for endergonic ET reactions.

These decay processes dominate over the dissociation of exciplexes into radical ions, especially at $\Delta G_{\mathrm{ET}}{ }^{*}>0$. In this region the quenching of the excited states actually occurs by partial ET with formation of transient exciplexes with $z<1$, which decay even in polar solvents predominantly by back ET (internal conversion or intersystem crossing), recovering parent ground states. Due to this fast internal conversion or intersystem crossing the quantum yield of radical ion formation decreases very rapidly with the increase of $\Delta G_{\mathrm{ET}}{ }^{*}[64,65]$. Thus, exciplex lifetime $\tau_{0}^{\prime}$ is controlled predominantly by the rates of internal conversion and intersystem crossing in exciplexes rather than by the rate of their dissociation into radical ions (especially at $\Delta G_{\mathrm{ET}}{ }^{*}>0$ ).

Thus, promoted radiationless decay can compete with exciplex formation (decreasing the exciplex yield), also reducing their lifetimes. Both these effects cause the increase of $k_{\mathrm{Q}}$ and decrease of radical ions yield.

\section{Competition of Different ET Mechanisms}

The overall ET mechanism can be revealed experimentally even in the absence of a transient exciplex emission by investigation of the dependence of $k_{\mathrm{Q}}$ on $\Delta G_{\mathrm{ET}}{ }^{*}$ and temperature dependence of $k_{\mathrm{Q}}$, especially. Several examples of $\log k_{\mathrm{Q}}$ versus $\Delta G_{\mathrm{ET}}{ }^{*}$ plots for various kinds of excited organic molecules and quenchers are shown in Figures 7, 8, $9,10,11,12,13,14$, and 15 , and parameters of these plots, according to the transient exciplex (13), and Marcus (3) and Weller (6) approaches, are given in Table 1. All the systems can be described by the transient exciplex model quite well and provide physically reasonable values of parameters 
TABle 1: Parameters of exciplex formation and decay, obtained from the dependences of $k_{Q}$ on $\Delta G_{E T}{ }^{*}(16)$ in various systems.

\begin{tabular}{|c|c|c|c|c|c|c|c|}
\hline Figure & System & $\log \tau_{0}^{\prime}+a / 2.3 R T$ & $a / \mathrm{eV}^{\mathrm{a}}\left(\log \tau_{0}^{\prime}\right)$ & $b / \mathrm{eV}$ & $c / \mathrm{eV}$ & $\lambda^{\mathrm{b}} / \mathrm{eV}$ & Reference \\
\hline 7 & $\begin{array}{l}\text { Various aromatics (in } \mathrm{MeCN} \text { ) } \\
\text { (charge separation) }\end{array}$ & -2.8 & $0.3(-8)$ & 0.06 & 0.3 & $\begin{array}{l}0.35 \\
0.11^{\mathrm{c}}\end{array}$ & {$[31]$} \\
\hline 8 & $\begin{array}{l}{ }^{1}(\mathrm{MeC}=\mathrm{O})_{2}{ }^{*} \text { and }{ }^{3}(\mathrm{MeC}=\mathrm{O})_{2} \\
+ \text { alkenes (in } \mathrm{MeCN})(\text { charge } \\
\text { separation) }\end{array}$ & -4.7 & $0.24(-9)$ & 0.35 & 0.28 & 0.9 & {$[33]$} \\
\hline 9 & $\begin{array}{l}\text { Oxonine }^{+}+\text {substituted benzenes } \\
\text { (in } \mathrm{MeOH}) \text { (charge shift) }\end{array}$ & -7.8 & $0.24(-12)$ & -0.05 & 0.28 & 0.3 & {$[43]$} \\
\hline 10 & $\begin{array}{l}{ }^{1} \text { Aromatics* }+ \text { chlorobenzenes (in } \\
\mathrm{MeCN}) \text { (charge separation) }\end{array}$ & -4.9 & $0.25(-9)$ & 0.32 & $\begin{array}{c}0.3 \\
(0.2-0.37) \\
\end{array}$ & 0.7 & {$[12]$} \\
\hline 11 & $\begin{array}{l}{ }^{3} \text { Quinones }+ \text { methylbenzenes } \\
\text { (in } \mathrm{MeCN} \text { and } \mathrm{CH}_{2} \mathrm{Cl}_{2} \text { ) } \\
\text { (charge separation) }\end{array}$ & -4.5 & $0.25(-9)$ & -0.22 & 0.2 & 0.1 & {$[14]$} \\
\hline 12 & $\begin{array}{l}{ }^{1} \text { Cyanoaromatics* }+ \text { methoxy- } \\
\text { benzenes and methylnaphthalenes } \\
\text { in } \mathrm{MeCN} \text { (charge separation) }\end{array}$ & -4.7 & $0.25(-8)$ & -0.23 & 0.2 & 0.25 & {$[15]$} \\
\hline \multirow[t]{2}{*}{13} & $\begin{array}{l}{ }^{1} 3 \text {-Carboxyethyl-7-methyl- } \\
\text { thioxanthen-9-one* + Methyl- } \\
\text { benzenes in } \mathrm{MeCN}\end{array}$ & -7.3 & $0.18(-11)$ & 0.13 & 0.1 & 0.1 & \multirow[t]{2}{*}{ [39] } \\
\hline & $\begin{array}{l}\text { + Methoxybenzenes in } \mathrm{MeCN} \\
\text { (charge separation) }\end{array}$ & -5.9 & $0.35(-11)$ & 0.13 & 0.1 & 0.5 & \\
\hline \multirow[t]{2}{*}{14} & $\begin{array}{l}{ }^{1} \text { Cyanoaromatics* }{ }^{*} \text { pyridine in } \\
\text { MeCN }\end{array}$ & -3.5 & $0.34(-10.5)$ & -0.05 & 0.5 & $<0$ & \multirow[t]{2}{*}[48]{} \\
\hline & $\begin{array}{l}\text { + Lutidine in MeCN (charge } \\
\text { separation) }\end{array}$ & -1.2 & $0.34(-8)$ & -0.05 & 0.5 & 0.1 & \\
\hline
\end{tabular}

${ }^{\mathrm{a}}$ Values of $a$ are obtained for the estimated values of $\log \tau_{0}^{\prime}$.

${ }^{\mathrm{b}}$ Values of $\lambda$ for (3) are estimated for $\Delta G_{\mathrm{ET}}{ }^{*}>-0.3 \mathrm{eV}$ (they are not valid at $\left.\Delta G_{\mathrm{ET}}{ }^{*}<-0.3 \mathrm{eV}\right)$. ${ }^{\mathrm{c}}$ Estimated values of $\Delta G_{0}^{\ddagger}$ for $(6)$.

$\left(\log \tau_{0}^{\prime}+a / 2.3 R T\right)=-(1-8), b=(-0.23)-(+0.35 \mathrm{eV})$, and $c=0.1-0.7 \mathrm{eV}$. Values of $\Delta G_{\mathrm{Ex}}$, obtained from the dependence of $k_{\mathrm{Q}}$ versus $\Delta G_{\mathrm{ET}}{ }^{*}$, are close to those, obtained by the direct measurements of $\Delta G_{\mathrm{Ex}}$ for various exciplexes in various solvents [38]. In the major part of the systems (see Figures 8-10, 13, 15) the difference between the dependences $\log k_{\mathrm{Q}}$ versus $\Delta G_{\mathrm{ET}} *$ according to different descriptions ((16), (3), (6)) is clear. Equations ((3), (6)) occur to describe experimental dependences only in the limited range of $\Delta G_{\mathrm{ET}}{ }^{*}$ (usually at $-0.3 \mathrm{eV}<\Delta G_{\mathrm{ET}}{ }^{*}<0.3 \mathrm{eV}$, see Table 1 and Figures 8-11, 15), and obtained reorganization energies are too small, relative to values, evaluated for long-range ET $(\lambda \approx 1-1.5 \mathrm{eV})$. For several systems (Figures 7 and 12 ) experimental data can be described with similar accuracy by all three equations. As matter of fact, the transient exciplex model at definite values of parameters provides dependence $\log k_{\mathrm{Q}}$ on $\Delta G_{\mathrm{ET}}{ }^{*}$, close to predicted by $((3)$, (6)) in experimentally studied range of $\log k_{\mathrm{Q}}$. But in many systems listed in the table transient exciplexes were observed experimentally by their emission spectra. For instance, in all the systems shown in Figure 12, the emission of transient exciplexes was observed, and the temperature dependences of both $\varphi_{0} / \varphi$ and $\varphi^{\prime} / \varphi((11),(12))$ provide equal values of parameters for quenching of excited molecules and exciplex formation and decay $[15,35-38,45]$. This gives reliable evidence for domination of the transient exciplex mechanism of quenching. One obtains too small values of $\lambda(<1 \mathrm{eV})$ or has to assume some shift of $\Delta G_{\mathrm{ET}}{ }^{*}$, if it tries to use (3) or (6) to describe the dependence of $\log k_{1}$ versus $\Delta G_{\mathrm{ET}}{ }^{*}$ for reaction, which actually follows the transient exciplex mechanism. The distinction between these plots for different mechanisms becomes obvious when $\tau_{0}^{\prime}<1 \mathrm{~ns}$, and one obtains $\lambda<0.5 \mathrm{eV}$ and a shift of $\Delta G_{\mathrm{ET}} *$ greater than $0.3 \mathrm{eV}$, when using (3) or (6).

More clear difference can be observed from the plots $\log \left(1 / k_{\mathrm{Q}}-1 / k_{1}\right)$ versus $\Delta G_{\mathrm{ET}}{ }^{*}$, which have different shapes, depending on the mechanism of ET. In the case of longrange ET mechanisms this plot should have parabolic form (or convex downwards hyperbolic form in the case of Weller's dependence (6)) and $k_{1} \approx k_{\text {Diff }}$

$$
\begin{aligned}
\log \left(\frac{1}{k_{\mathrm{Q}}}-\frac{1}{k_{\text {Diff }}}\right)= & -\log \left(K_{\text {Ass }} k^{0}\right)+\frac{\Delta G^{\ddagger}}{2.3 R T} \\
= & -\log \left(K_{\text {Ass }} k^{0}\right)-\log \sum_{m}\left\{\left[\frac{\exp (-S) S^{m}}{m !}\right]\right. \\
& \left.\times \exp \left[-\frac{\left(\Delta E-m h \nu_{V}\right)^{2}}{4 \lambda_{S} k_{B} T}\right]\right\},
\end{aligned}
$$

where $\log \left(K_{\text {Ass }} k^{0}\right) \approx 10-12$ and $h \nu_{V} \approx 0.1-0.2 \mathrm{eV}, m \geq 0$. In the case of transient exciplex mechanism the dependence of $\Delta G_{\mathrm{Ex}}{ }^{*}$ on $\Delta G_{\mathrm{ET}}{ }^{*}$ is close to a convex upwards hyperbolic 


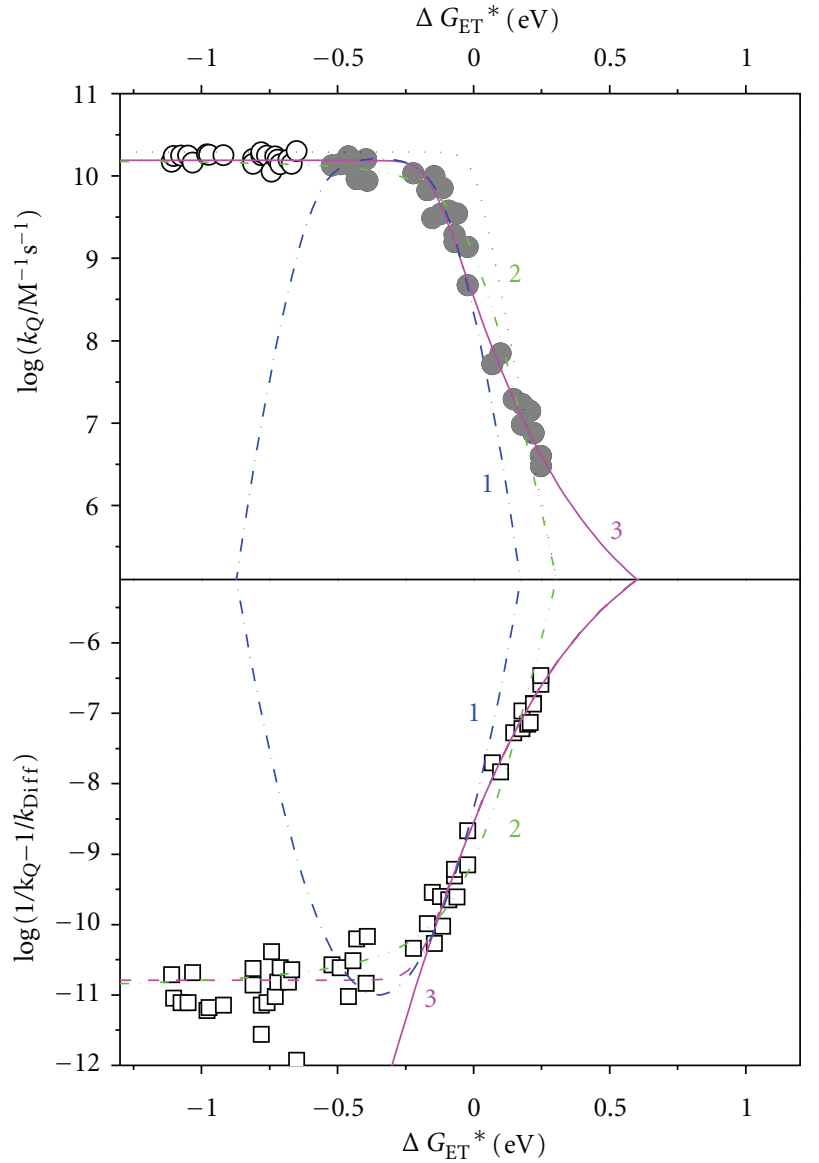

Figure 7: Dependences of $\log k_{\mathrm{Q}}$ (top) and $\log \left(1 / k_{\mathrm{Q}}-1 / k_{\text {Diff }}\right)$ (bottom) on $\Delta G_{\mathrm{ET}}$ * for quenching of excited singlet states of various aromatic compounds in $\mathrm{MeCN}$ (according to the experimental data of Rehm and Weller [31]). Best plots are shown for simulation of the experimental dependence by (3) -1 and (6) -2 , and by concatenated mechanism (16) - 3. Dotted line shows thermodynamic limit for ET $\left(k_{\mathrm{Q}}=k_{\text {Diff }} /\left[1+k^{0} \exp \left(-\Delta G_{\mathrm{ET}} * / 2.3 R T\right)\right]\right.$, where $\log k_{\text {Diff }}=$ $\left.10.3, \log k^{0}=13\right)$.

curve and the plot $\log \left(1 / k_{\mathrm{Q}}-1 / k_{1}\right)$ versus $\Delta G_{\mathrm{ET}}{ }^{*}$ has a form close to hyperbolic (with the orientation opposite to the Weller's dependence) and can be approximated as

$$
\begin{aligned}
& \log \left(\frac{1}{k_{\mathrm{Q}}}-\frac{1}{k_{1}}\right) \\
& =\log \tau_{0}^{\prime}+\frac{\Delta G_{\mathrm{Ex}}{ }^{*}}{2.3 R T} \\
& =\log \tau_{0}^{\prime}+\frac{\left\{a+\left(\Delta G_{\mathrm{ET}} *+b\right) / 2-\left[\left(\Delta G_{\mathrm{ET}} *+b\right)^{2} / 4+c^{2}\right]^{1 / 2}\right\}}{2.3 R T} .
\end{aligned}
$$

However, it must take into account that actually these experimental dependences are cut off by the horizontal limit near $\log \left(1 / k_{\mathrm{Q}}-1 / k_{1}\right)=-(10-11)$ and have sigmoid shape since one has to use some overestimated values for $k_{1}$ to prevent too large scattering and negative values of $\left(1 / k_{\mathrm{Q}}-1 / k_{1}\right)$,



Figure 8: Dependences of $\log k_{\mathrm{Q}}$ (top) and $\log \left(1 / k_{\mathrm{Q}}-1 / k_{\text {Diff }}\right)$ (bottom) on $\Delta G_{\mathrm{ET}}{ }^{*}$ for quenching of excited singlet (1) and triplet (2) states of biacetyl by alkenes in $\mathrm{MeCN}$ (according to the experimental data of Gersdrof et al. [33]). Best plots are shown for simulation of the experimental dependence by (3) $(\lambda=0.9 \mathrm{eV})$ (dash line) and by concatenated mechanism (16) (solid line). Dotted line shows thermodynamic limit for ET.

arising due to some variations $k_{\text {Diff }}$ and $k_{1}$ for different compounds $\left(\log k_{1} \approx 10.1-10.3\right)$.

Usually the dependence of $k_{\mathrm{Q}}$ on $\Delta G_{\mathrm{ET}} *$ for similar compounds can be described by the same values of these parameters (see Figures 7-12 and lines 7-12 in the Table). In many cases (see Figures 8-11, 13, 14) experimentally obtained $k_{\mathrm{Q}}$ even exceeds thermodynamic limit for complete electron transfer $\left(\log k_{\mathrm{ET}}=-\log \left[1 / k_{\text {Diff }}+\exp \left(\ln \left(k_{\mathrm{B}} T / h\right)+\right.\right.\right.$ $\left.\left.\Delta G_{\mathrm{ET}}{ }^{*} / R T\right)\right]$ ) since transient exciplexes decay predominantly by internal conversion and intersystem crossing rather than by dissociation into radical ions $\left(\tau_{0}^{\prime} \ll 1 / k_{\mathrm{R}}^{\prime}\right)$. Large scattering of experimental $\log k_{\mathrm{Q}}$ observed in some systems (see, e.g., Figure 10) can be attributed to variations of $V_{\mathrm{AD}}(\approx$ $c=0.2-0.4 \mathrm{eV})$ as well as to variations of $\tau_{0}^{\prime}$ for these exciplexes. Even small variations of the parameters in (11) can cause essential effect on the shape of the dependence $\log k_{\mathrm{Q}}$ versus $\Delta G_{\mathrm{ET}}{ }^{*}$. Experimental pieces of evidence for strong variations of $\Delta G_{\mathrm{Ex}}{ }^{*}$ and $\tau_{0}^{\prime}$ for exciplexes of very similar compounds are known $[38,48]$. These variations 


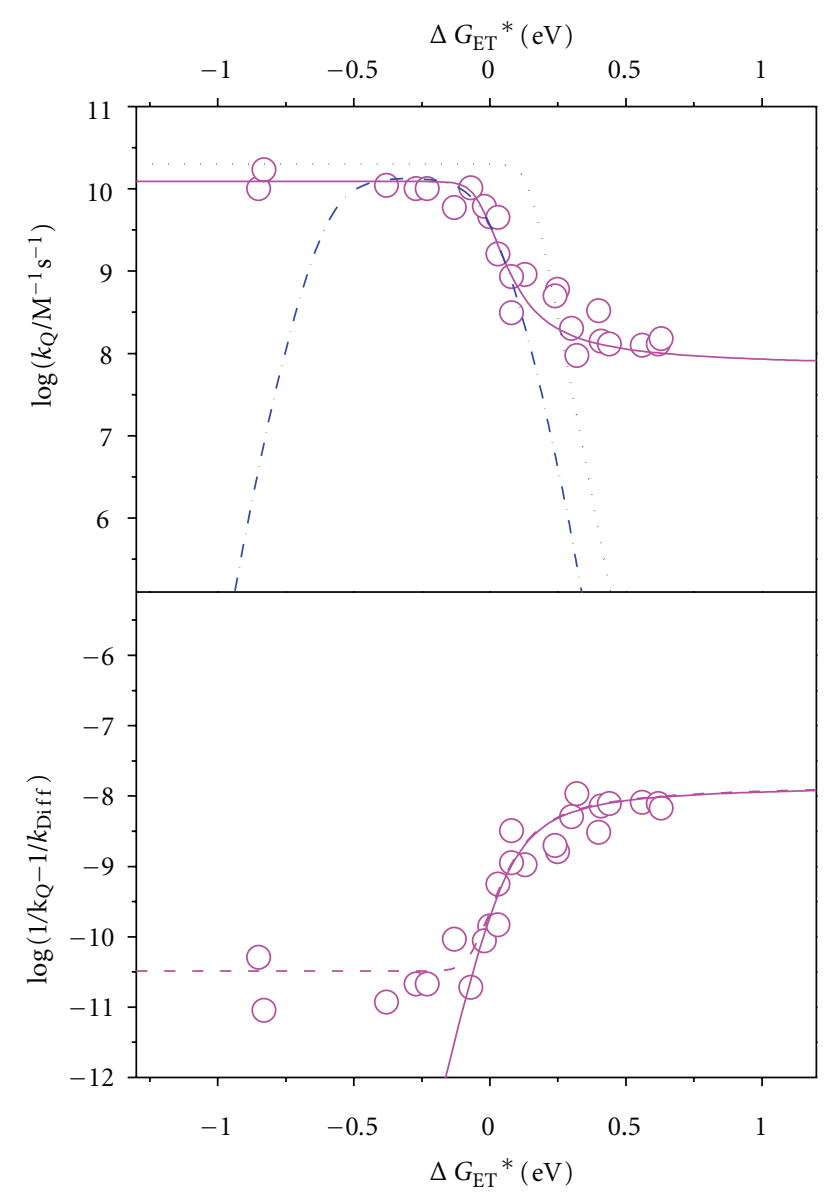

Figure 9: Dependences of $\log k_{\mathrm{Q}}$ (top) and $\log \left(1 / k_{\mathrm{Q}}-1 / k_{\text {Diff }}\right)$ (bottom) on $\Delta G_{\mathrm{ET}}$ * for quenching of excited singlet state of oxonine cation by various aromatic compounds in $\mathrm{MeOH}$ (according to the experimental data of [43]). Best plots are shown for simulation of the experimental dependence by $(3)(\lambda=0.3 \mathrm{eV})$ (dash line) and by concatenated mechanism (16) (solid line). Dotted line shows thermodynamic limit for ET.

were found to be responsible for marked distinctions in plots of $\log k_{\mathrm{Q}}$ versus $\Delta G_{\mathrm{ET}}{ }^{*}$, rather than a difference in ET mechanisms for such compounds. For instance, different dependencies of $k_{\mathrm{Q}}$ versus $\Delta G_{\mathrm{ET}}{ }^{*}$ were observed for quenching of an excited singlet thioxantone in $\mathrm{MeCN}$ (Figure 13) [39] by methylbenzenes and methoxybenzenes (and some other steric hindered quenchers) in spite of negligible solvent polarity effect, found for both kinds of the quenchers. At the same time exciplex emission frequencies for exciplexes of methylbenzenes are substantially (ca. $0.1 \mathrm{eV}$ ) smaller than those for exciplexes of methoxybenzenes and steric hindered compounds (Figure 13). This indicates more negative $\Delta G_{\mathrm{ET}}{ }^{*}$ for exciplexes of methylbenzenes than those for similar exciplexes of methoxybenzenes due to steric hindrance. Both, more negative $\Delta G_{\mathrm{Ex}}{ }^{*}$ and shorter lifetime of exciplexes of methylbenzenes, are responsible for the observed difference in experimental plots of $\log k_{\mathrm{Q}}$ versus $\Delta G_{\mathrm{ET}}{ }^{*}$.

Even stronger difference in the dependence of $k_{\mathrm{Q}}$ on $\Delta G_{\mathrm{ET}}{ }^{*}$ for quite similar compounds was observed in the

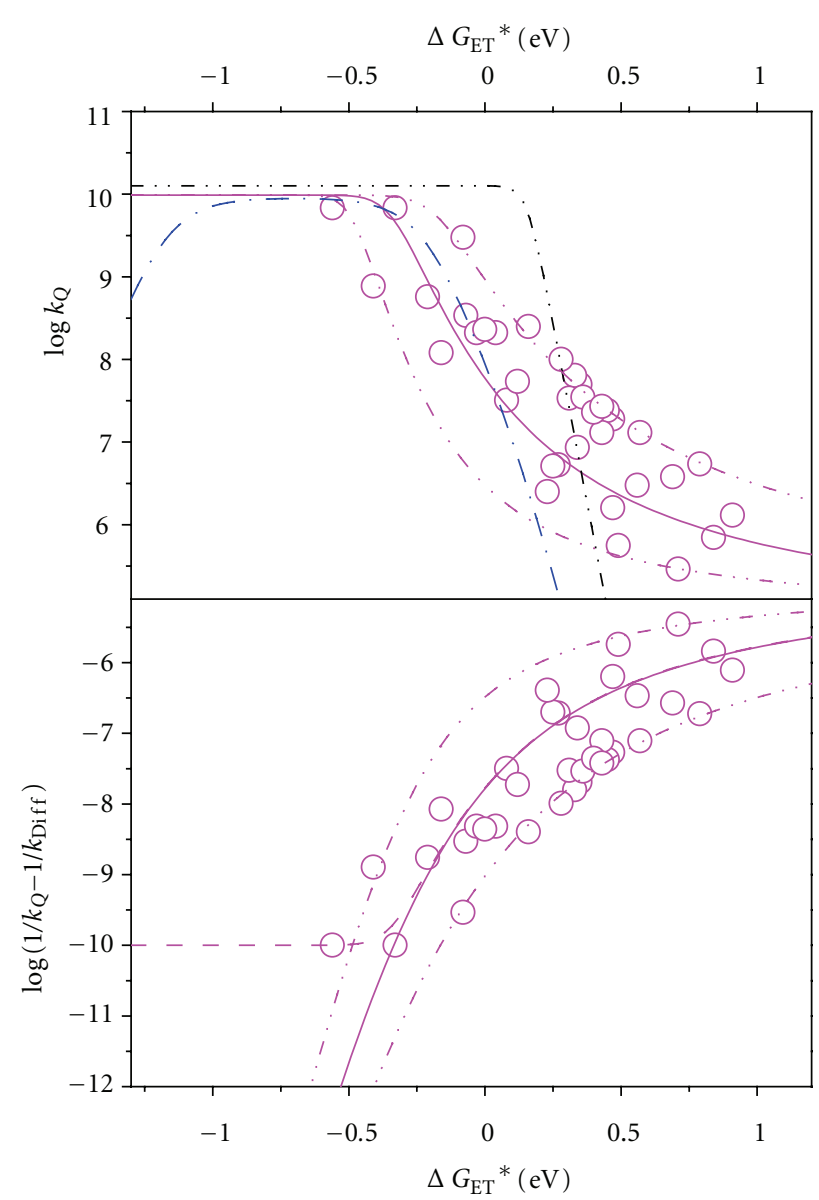

FIGURE 10: Dependences of $\log k_{\mathrm{Q}}$ (top) and $\log \left(1 / k_{\mathrm{Q}}-1 / k_{\text {Diff }}\right)$ (bottom) on $\Delta G_{\mathrm{ET}}$ * for quenching of excited singlet states of various aromatic compounds by chlorobenzenes in $\mathrm{MeCN}$ (according to the experimental data of Grosso et al. [12]). Best plots are shown for simulation of the experimental dependence by $(3)(\lambda=0.7 \mathrm{eV})$ (dash line) and by concatenated mechanism (16) (solid line). Dotted line shows thermodynamic limit for ET.

case of quenching of excited cyanoaromatics by pyridine and lutidine (2, 6-dimethylpyridine) in MeCN by Wang et al. [48] (Figure 14). Both pyridine and lutidine quench efficiently cyanoaromatics excited states but for pyridine $k_{\mathrm{Q}}$ drops at much greater endergonicity $\left(\Delta G_{\mathrm{ET}}{ }^{*}>0.5 \mathrm{eV}\right)$ in spite of rather small difference of redox potentials of pyridine and lutidine (2.62 and $2.37 \mathrm{eV}$ versus SCE, resp.). In terms of the concatenated mechanism this difference can be attributed to the strong difference of $\tau_{0}^{\prime}$ for pyridine and lutidine exciplexes. Actually, lutidine forms fluorescing exciplexes with cyanoaromatics ( $\tau_{0}^{\prime}$ is ca. $5 \mathrm{~ns}$ ) but pyridine exhibits no exciplex fluorescence even in low-polar solvents. Very fast $(<60 \mathrm{ps})$ recovery of the absorption spectrum of parent cyanoaromatics observed in neat pyridine [48] confirms very short pyridine exciplex lifetime.

So short pyridine exciplex lifetime in contrast to the lutidine exciplex can be attributed to the presence of weak ionogenic $\mathrm{C}-\mathrm{H}$ bonds in 2 and 6 positions in the pyridine radical cation fragment of the exciplex in contrast to the 


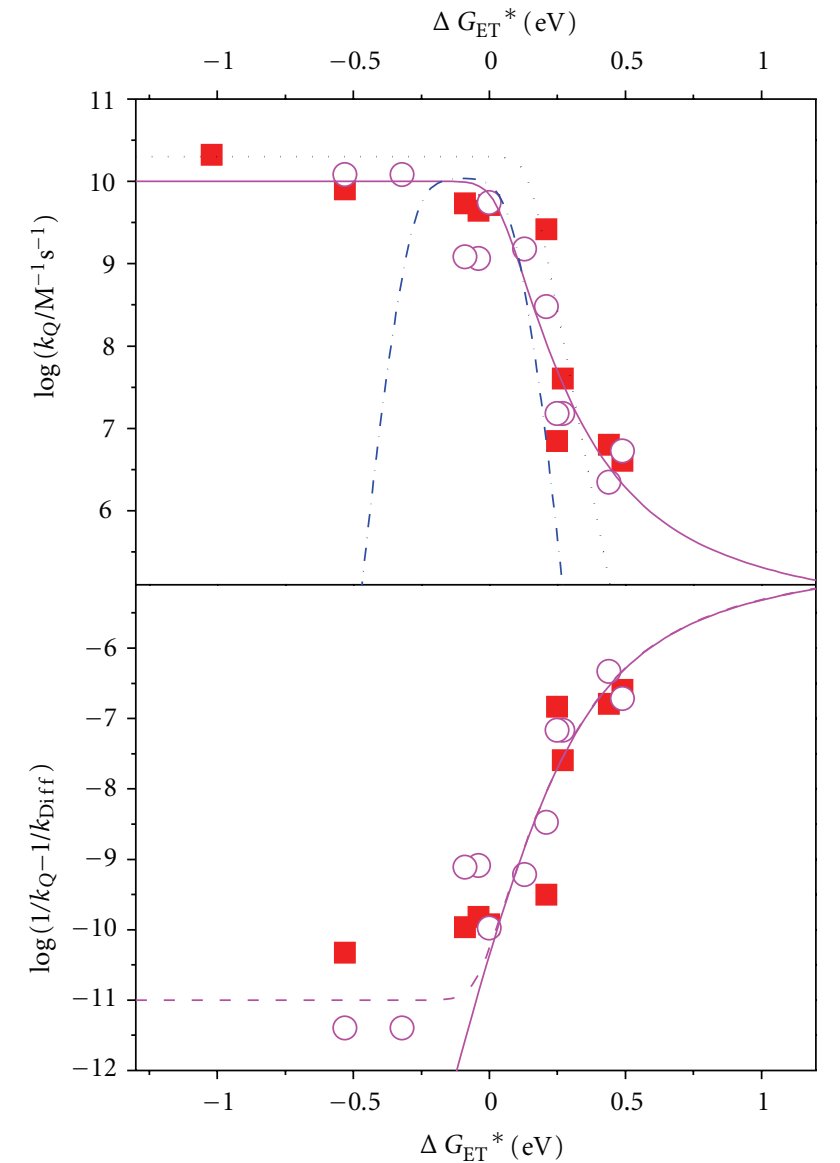

Figure 11: Dependences of $\log k_{\mathrm{Q}}$ (top) and $\log \left(1 / k_{\mathrm{Q}}-1 / k_{\text {Diff }}\right)$ (bottom) on $\Delta G_{\mathrm{ET}} *$ for quenching of triplet states of various quinones by methylbenzenes in $\mathrm{MeCN}$ (circles) and $\mathrm{CH}_{2} \mathrm{Cl}_{2}$ (squares) (according to the experimental data of Hubig and Kochi [14]). Best plots are shown for simulation of the experimental dependence by (3) $(\lambda=0.1 \mathrm{eV})$ (dash line) and by concatenated mechanism (16) (solid line). Dotted line shows thermodynamic limit for ET.

lutidine exciplex where these positions are substituted by methyl groups. Some similarity can be mentioned between the exciplexes of lutidine and tertiary amines in contrast to exciplexes of pyridine and primary and secondary amines which show no emission. In both cases weak $\mathrm{C}-\mathrm{H}$ or $\mathrm{N}-\mathrm{H}$ bonds in the radical cation fragment of the exciplexes promote very fast radiationless decay (see previous section). wang et al. invented another very interesting hypothesis of the transient formation of "bonded exciplexes" [48] to explain the obtained results. This hypothesis implies the formation of short-lived transients with a $\sigma$-bond between nitrogen atom of pyridine and carbon atom of the aromatic nucleus of cyanoaromatic compound. But this hypothesis contradicts very fast (ca. $60 \mathrm{ps}$ ) recovering of the parent dicyanoanthracene [48], since formation and dissociation of $\sigma$-bond $\mathrm{C}-\mathrm{N}$ are expected to have substantial activation energy. Besides, the formation of this kind of a transient in ET reaction should have either activation energy strongly depending on $\Delta G_{\mathrm{ET}}{ }^{*}\left(\Delta G^{\ddagger} \gg 0.2 \mathrm{eV}\right.$ at $\Delta G_{\mathrm{ET}}{ }^{*}>0.5 \mathrm{eV}$

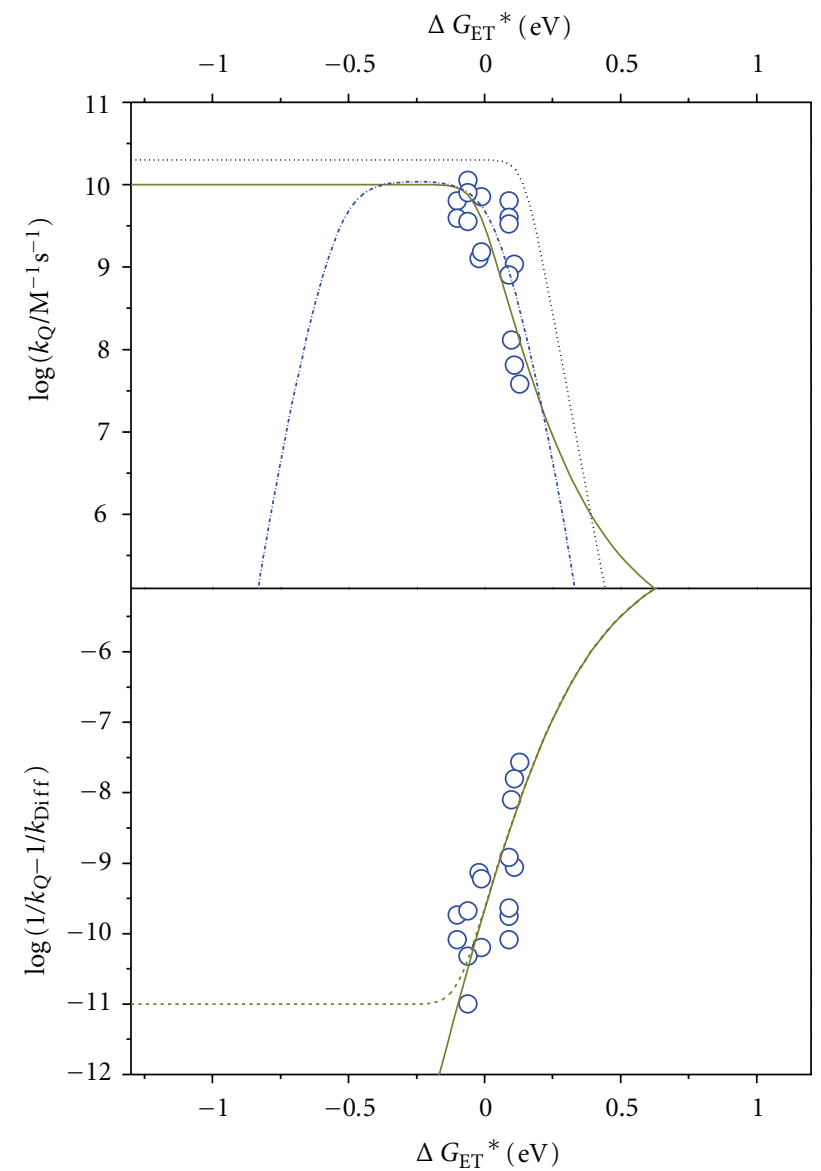

Figure 12: Dependences of $\log k_{\mathrm{Q}}$ (top) and $\log \left(1 / k_{\mathrm{Q}}-1 / k_{\text {Diff }}\right)$ (bottom) on $\Delta G_{\mathrm{ET}}{ }^{*}$ for quenching of excited singlet states of cyanoaromatic compounds by methoxybenzenes and methylnaphthalenes in $\mathrm{MeCN}$ (according to the experimental data [15]). Best plots are shown for simulation of the experimental dependence by (3) $(\lambda=0.25 \mathrm{eV})$ (dash line) and by concatenated mechanism (16) (solid line). Dotted line shows thermodynamic limit for ET.

and $\Delta G^{\ddagger}<0.2 \mathrm{eV}$ at $\Delta G_{\mathrm{ET}}{ }^{*}<0.5 \mathrm{eV}$ ), or transient should have rather long lifetime $\left(>10^{-8} \mathrm{~s}\right)$ to explain experimentally observed quenching rate constants $k_{\mathrm{Q}}<10^{7} \mathrm{M}^{-1} \mathrm{~s}^{-1}$ at $\Delta G_{\mathrm{ET}}{ }^{*}>0.6 \mathrm{eV}$. In contrast, transient exciplex mechanism requires only substantial values of the electronic coupling matrix element (ca. $0.5 \mathrm{eV}$ ) and large difference of the transient exciplex lifetimes, related to specific chemical behavior of pyridine (presence of $\mathrm{H}$ atoms in $\alpha$ position, promoting very fast radiationless decay of an exciplex).

Thus, even small variations of the structure of a quencher or excited molecules cause strong changes of $k_{\mathrm{Q}}$. All these cases find their natural explanation in terms of the transient exciplex mechanism. High values of $k_{\mathrm{Q}}$ for pyridine (close to the diffusion controlled values even when $\Delta G_{\mathrm{ET}}{ }^{*}$ reaches $0.5 \mathrm{eV}$ ) confirm that reaction of exciplex formation has no essential energy barrier except that for diffusion.

Very small effect of the medium polarity on $k_{\mathrm{Q}}$, observed for majority of quenchers, confirms the transient exciplex mechanism. Exciplex solvation energy proportional 


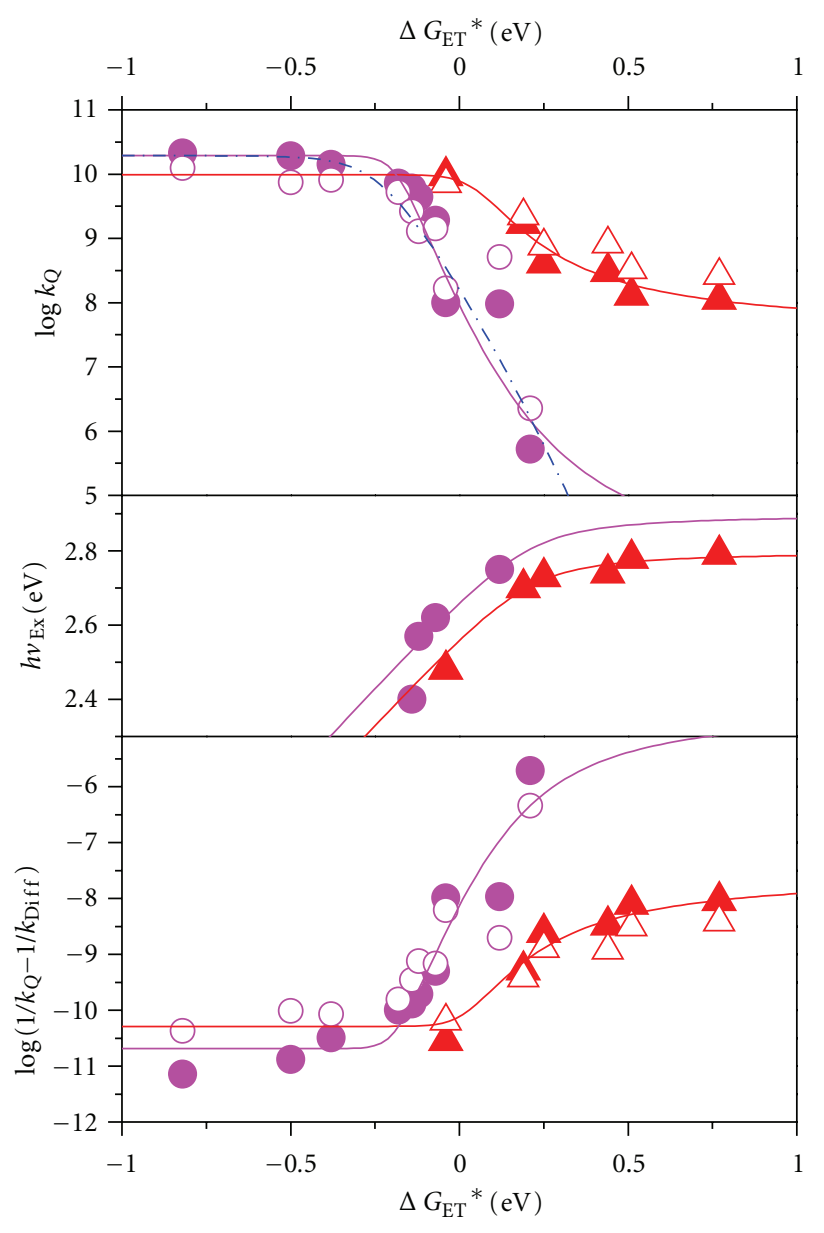

Figure 13: Dependences of $\log k_{\mathrm{Q}}$ (top) and $\left.\log \left(1 / k_{\mathrm{Q}}-1 / k_{\text {Diff }}\right)\right)$ (bottom) on $\Delta G_{\mathrm{ET}}{ }^{*}$ for quenching of excited singlet state of thioxanthone by methylbenzenes (triangles) and methoxybenzenes (circles) in MeCN (according to the experimental data of Jacques et al. [39]). Best plots are shown for simulation of the experimental dependence by (3) ( $\lambda=0.1$ and $0.5 \mathrm{eV}$, resp.) (dash lines) and by concatenated mechanism (16) (solid lines). Dotted line shows thermodynamic limit for ET. Experimental dependence of the exciplex emission frequencies, $h \nu_{\mathrm{Ex}}$, on $\Delta G_{\mathrm{ET}}{ }^{*}$ in $\mathrm{MeCN}$ is shown in the middle panel.

to $\left(\mu_{\mathrm{Ex}}{ }^{2} / \rho^{3}\right) \mathrm{f}(\varepsilon)$ depends on $\varepsilon$ much weaker than Marcus reorganization energy (see (3)). Sometimes an investigation of the temperature dependence of $\log k_{Q}$ (determination of apparent $\Delta H_{\mathrm{Q}}^{\ddagger}$ ) is necessary to distinguish the transient exciplex and long-range mechanisms.

\section{Main Features and Paradoxes of Excited-State ET Reactions}

Several features of the excited-state ET reactions, contradicting common long-range mechanism of ET, are well known [30]. These paradoxes find natural explanation in terms of transient exciplex formation mechanism.

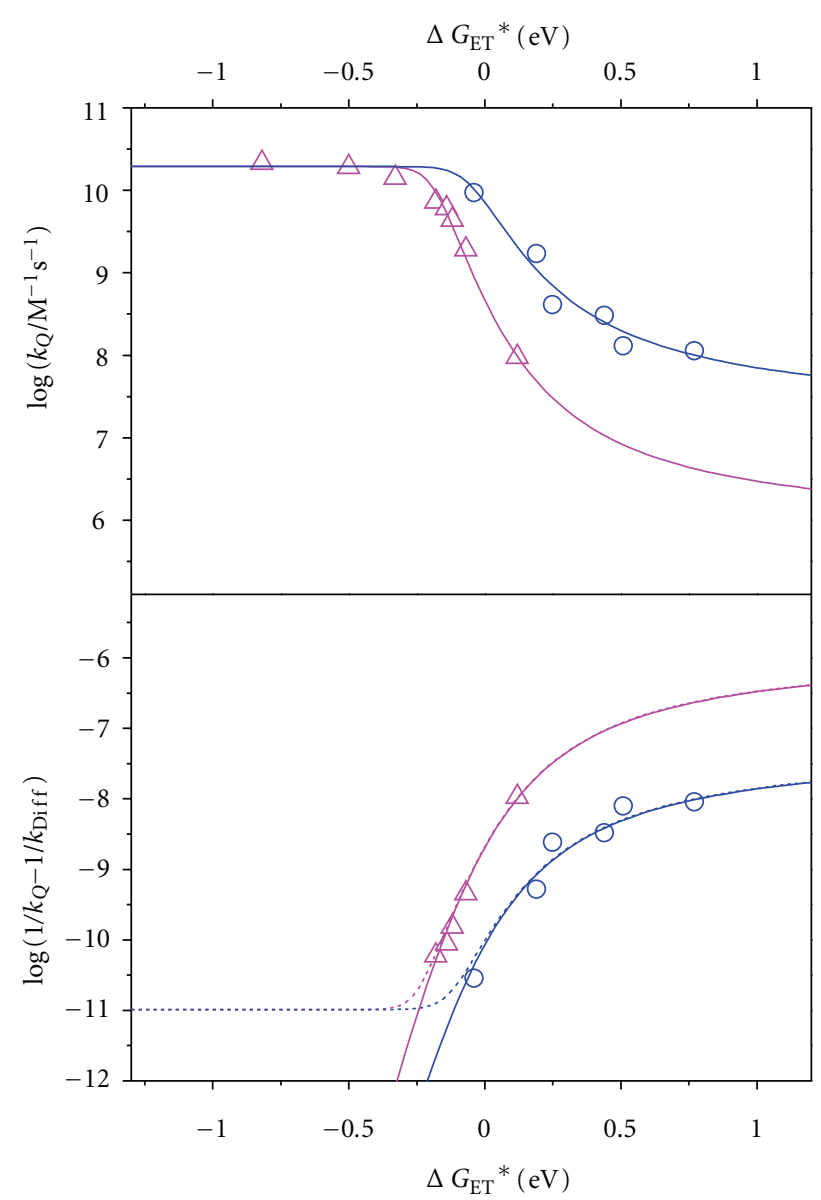

Figure 14: Common dependence of $\log k_{\mathrm{Q}}$ (top) and $\log \left(1 / k_{\mathrm{Q}}-\right.$ $1 / k_{\text {Diff }}$ ) (bottom) on $\Delta G_{\mathrm{Ex}}{ }^{*}$ (obtained according to (16)) of exciplexes of thioxantone with methylbenzenes (circles) and methoxybenzenes (squares) in $\mathrm{MeCN}$ (according to the experimental data of Jacques et al. [39]). Lifetimes of transient exciplexes were supposed to be $10 \mathrm{ps}$ for methylbenzenes and $300 \mathrm{ps}$ for methoxybenzenes.

(1) Absence of the exciplex emission cannot testify against the transient exciplex formation since many exciplexes have much smaller emission rate constants, $k_{\mathrm{F}^{\prime}}$, and lifetimes, $\tau_{0}^{\prime}$, relative to parent excited molecules. Besides, exciplex emission spectra can overlap significantly with parent reactant fluorescence, especially when $\Delta G_{\mathrm{ET}} *>0$, and special ingenuity is often necessary to reveal them. Radiative transitions in exciplexes are forbidden by symmetry and $k_{\mathrm{F}^{\prime}}$ is usually proportional to the contribution of the locally excited state $(1-z)[66,72]$. Exciplex lifetimes can be very short because of efficient radiationless decay (internal conversion into the ground state and intersystem crossing into the triplet state) $[28,45]$ (see, Section 3$)$.

(2) Nonexponential fluorescence decay of parent $M^{*}$ is frequently observed because of reversible exciplex formation $\left(\mathrm{M}^{*}+\mathrm{Q} \rightleftarrows \mathrm{Ex}\right)$. In such a case the decay of $\mathrm{M}^{*}$ is biexponential $[19,66]$

$$
I(t)=a \exp \left(-\frac{t}{\tau_{1}}\right)+(1-a) \exp \left(-\frac{t}{\tau_{2}}\right)
$$




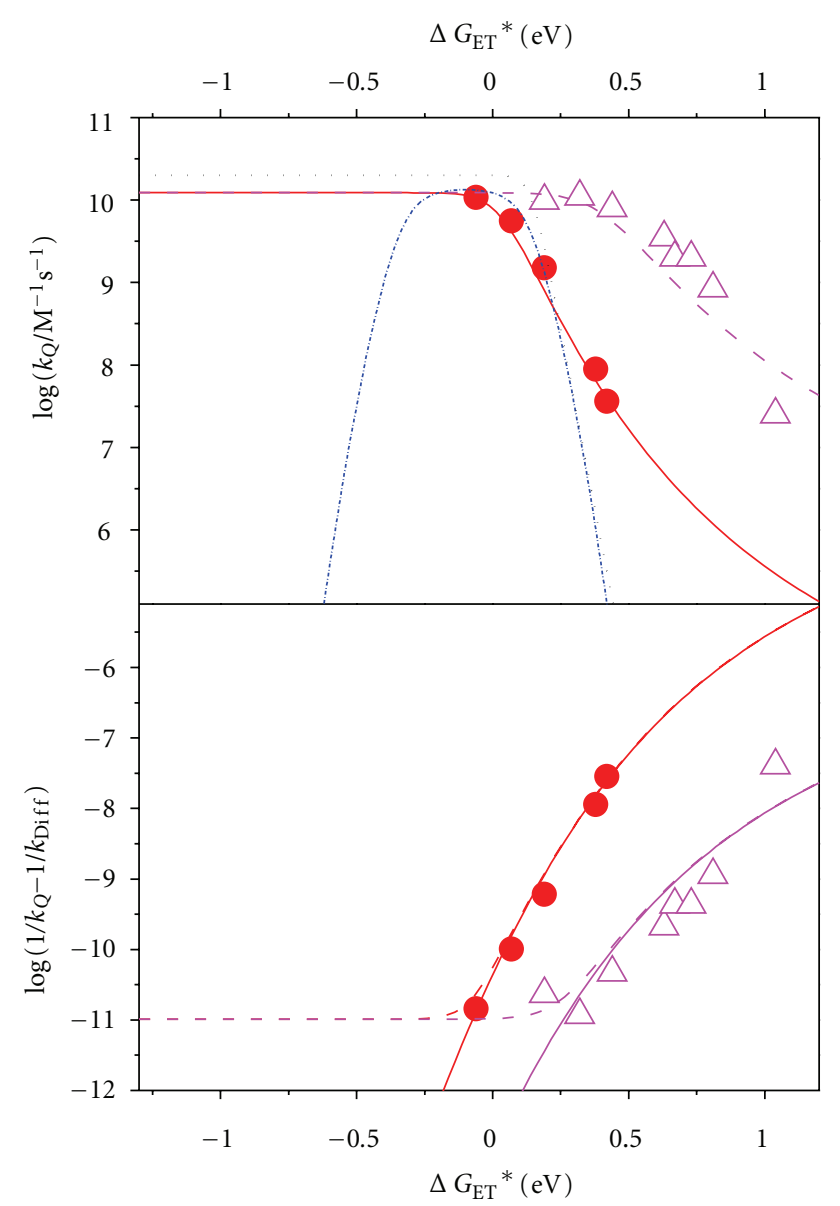

Figure 15: Dependences of $\log k_{\mathrm{Q}}$ (top) and $\log \left(1 / k_{\mathrm{Q}}-1 / k_{\text {Diff }}\right)$ (bottom) on $\Delta G_{\mathrm{ET}}{ }^{*}$ for quenching of singlet excited states of cyanoaromatic compounds by pyridine (triangles) and lutidine (circles) in MeCN (according to the experimental data of Wang et al. [48]). Best plots are shown for simulation of the experimental dependence by concatenated mechanism (16) (solid lines) and by (3) (lutidine, $\lambda=0.1 \mathrm{eV}$ ) (dash line). Dotted line shows thermodynamic limit for ET.

where decay rate constants $1 / \tau_{1,2}$ depend on the quencher concentration nonlinearly

$$
\begin{aligned}
\frac{1}{\tau_{1,2}}= & \frac{\left(1 / \tau_{0}+k_{1}[\mathrm{Q}]+1 / \tau_{0}^{\prime}+k_{-1}\right)}{2} \\
& \pm\left\{\frac{\left(1 / \tau_{0}+k_{1}[\mathrm{Q}]-1 / \tau_{0}^{\prime}-k_{-1}\right)^{2}}{4}+k_{-1} k_{1}[\mathrm{Q}]\right\}^{1 / 2} .
\end{aligned}
$$

It should be mentioned that frequently only one of these exponents can be observed experimentally because of strong difference in their amplitudes or decay times. For instance, quenching of anthracene fluorescence by $\mathrm{N}, \mathrm{N},-$ dimethylaniline [66] demonstrates substantially greater slope of the plot $\tau_{0} / \tau_{1}$ versus $[\mathrm{Q}]$ relative to $\varphi_{0} / \varphi$ versus [Q] and almost constant $\tau_{0} / \tau_{2} \ll \tau_{0} / \tau_{1}$. But in the case of pyrene [66] $\tau_{1}$ is several hundred times smaller than $\tau_{2}$ and can be missed

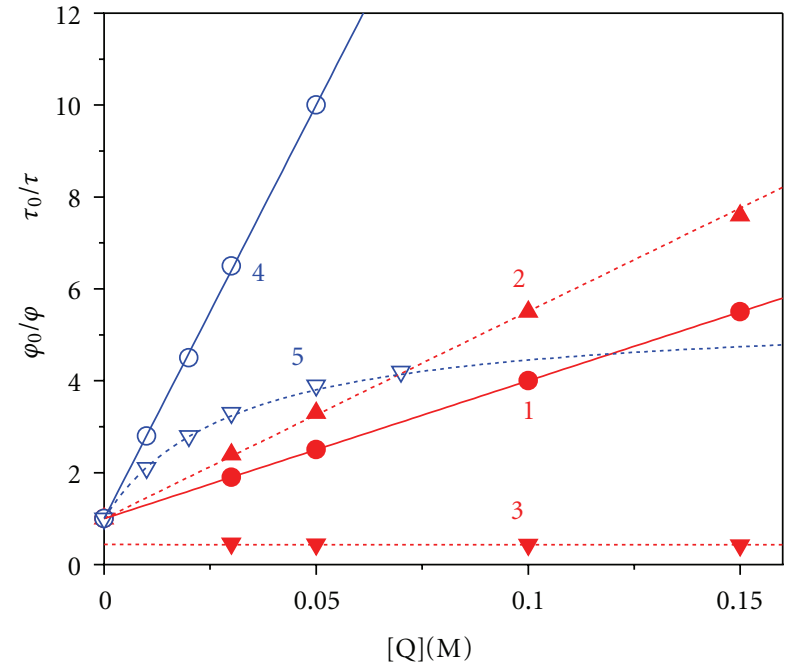

Figure 16: Dependences of $\varphi_{0} / \varphi(1,4)$ and $\tau_{0} / \tau_{1,2}(2,3,5)$ on the concentration of $\mathrm{N}, \mathrm{N}$-diethylaniline in heptane for anthracene ( 1 , $2,3)$ and pyrene $(4,5)$ [49].

in actual measurements in spite of its greater amplitude. In all the cases $\tau_{0} / \tau_{1}>\varphi_{0} / \varphi>\tau_{0} / \tau_{2}$.

(3) Sublinear dependence of $\tau_{0} / \tau$ on the quencher concentration (Figure 16) and deviations from Stern-Volmer equation are observed based on the same reason [49] in contrast to the linear dependence of fluorescence quantum yields $\left(\varphi_{0} / \varphi=1+k_{1} \tau_{0}[\mathrm{Q}] /\left(1+k_{-1} \tau_{0}^{\prime}\right)\right)$.

(4) Non-Arrhenius dependence of $K_{\mathrm{SV}}=\left(\varphi_{0} / \varphi-\right.$ 1)/[Q] on reciprocal temperature, 1/T, (Figure 17) which contradicts activated behavior of ET, is frequently observed because exciplex formation is controlled by the diffusion at low temperatures and by thermodynamics $\left(\Delta H_{\mathrm{Ex}}{ }^{*}\right)$ of exciplex formation at high temperatures $[44,45]$. Based on this reason this temperature dependence has a bell shape ((11), (12)). Negative slope of the low temperature wing $\left(k_{-1} \tau_{0}^{\prime} \ll 1\right)$ is equal to $-\Delta H_{1}^{\ddagger} / R T \approx-\Delta H_{\text {Diff }}^{\ddagger} / R T$, but the slope of high temperature wing $\left(k_{-1} \tau_{0}^{\prime} \gg 1\right)$ is equal to $-\left(\Delta H_{\mathrm{Ex}}{ }^{\ddagger}-\Delta H_{\mathrm{Ex}}{ }^{*}\right) / R$.

Negative values of apparent activation energy of quenching $\Delta H_{\mathrm{Q}}^{\ddagger}$ (obtained from the plot $\ln \left(\varphi_{0} / \varphi-1\right) / \tau_{0}[\mathrm{Q}] \approx$ $\ln k_{\mathrm{Q}}^{0}+\Delta S_{\mathrm{Q}}^{\ddagger} / R-\Delta H_{\mathrm{Q}}^{\ddagger} / R T$ ) observed in many cases (Figure 18) $[67-70,73,74]$ arise when $T\left(\Delta S_{-1}^{\ddagger}-\Delta S_{\text {Ex }}^{\ddagger}\right)>\left(\Delta H_{-1}^{\ddagger}-\right.$ $\left.\Delta H_{\mathrm{Ex}}^{\ddagger}\right)$ and exciplexes decay predominantly by radiationless mechanism $\left(\Delta H_{\mathrm{Ex}}^{\ddagger} \approx 0\right)[15,44-46]$.

(5) Weak solvent polarity effect is observed frequently for quenching rate constants $[38,67,73,74]$ (see, for instance, Figure 11) in contrast to the expected strong effect of $\varepsilon$ on $\lambda$ according to (4). It can be attributed to small polarity of transient exciplexes relative to radical ion pairs $\left(\mu_{\mathrm{Ex}} \ll \mu_{\mathrm{RIP}}\right)$, when $\Delta G_{\mathrm{ET}}{ }^{*}>0$, and weak influence of solvent polarity on $\Delta G_{\mathrm{Ex}}{ }^{*}$. This effect is expected when radiationless decay of transient exciplex dominates over its dissociation into radical ions.

(6) Quenching of singlet excited states is not frequently followed by the decrease of the triplet state yield (Figure 18). 




FIGURE 17: Experimental apparent activation free energy (blue $\square$ ) and activation enthalpy (red $\bigcirc$ ) for quenching of aromatic compounds by various quenchers [66-71]. Free energies of transient exciplex formation (magenta $\boldsymbol{\Delta}$ ) are calculated according to (10) (for $\tau_{0}^{\prime}=1 \mathrm{~ns}$ ); the entropy of transient exciplex formation (green $\downarrow$ ) $T \Delta S_{\mathrm{Ex}}{ }^{*}=\Delta H_{\mathrm{Ex}}{ }^{*}-\Delta G_{\mathrm{Ex}}{ }^{*}$ (assuming $\Delta H_{\mathrm{Ex}}{ }^{*} \approx \Delta H_{\mathrm{App}}^{\ddagger}$ ). Simulated curves are: (1) $\Delta G^{\ddagger}$ for Marcus mechanism ((2), $\lambda=$ $0.6 \mathrm{eV})$ and (2) $\Delta G_{\mathrm{Ex}}{ }^{*}$ for transient exciplex mechanism ((15), $a=$ $0.13, b=0.07, c=0.14 \mathrm{eV})$; (3) $\Delta H_{\mathrm{App}}^{\ddagger}=0.12-\Delta G_{\mathrm{ET}} * / 2-$ $\left(\left(\Delta G_{\mathrm{ET}} * / 2\right)^{2}+0.14^{2}\right)^{1 / 2}$.

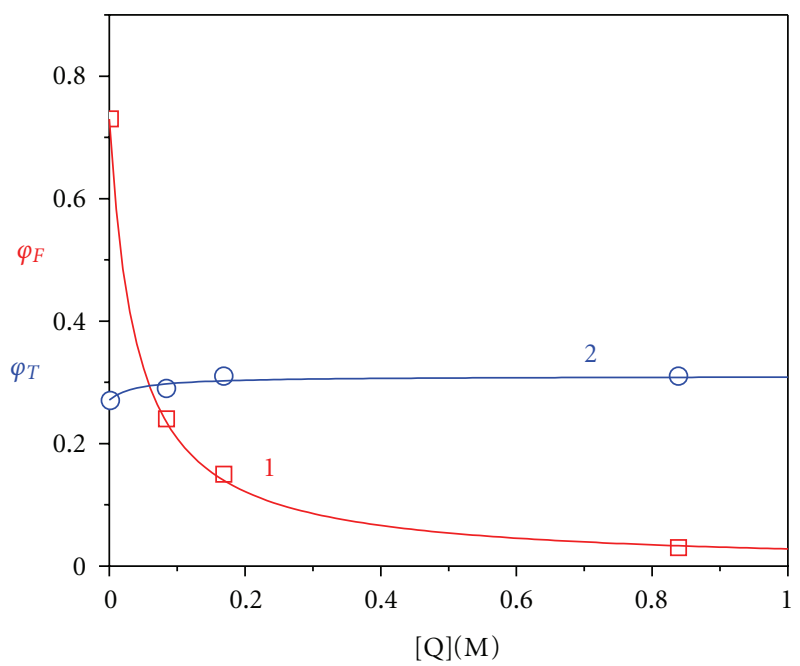

FIGURE 18: Dependences of pyrene fluorescence and triplet state quantum yields on the concentration of dibutyl phthalate in acetonitrile at room temperature [69].

Sometimes this quenching is followed even by the increase of the triplet yield $[67,75]$. This contradicts the long-range ET mechanism but is quite natural for transient exciplex mechanism since exciplex decay occurs by both internal conversion and intersystem crossing and can provide rather high quantum yield of triplets. When the ratio of intersystem crossing $\left(k_{\mathrm{ISC}}\right)$ and internal conversion $\left(k_{\mathrm{IC}}\right)$ rate constants in the exciplex $\left(k_{\mathrm{ISC}}^{\prime} / k_{\mathrm{IC}}^{\prime}\right)$ is higher than this ratio in parent

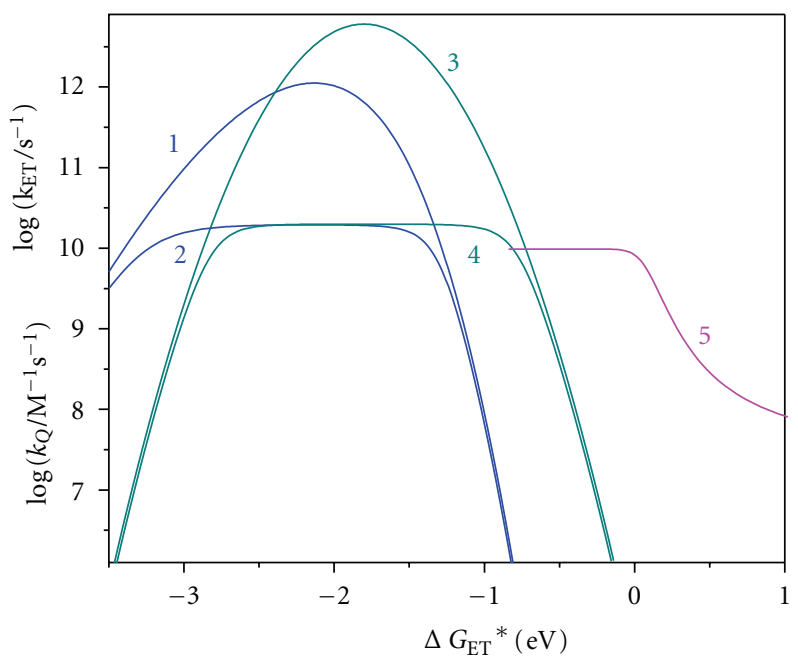

Figure 19: Simulated dependences of unimolecular $(1,3)$ rate constants of excited-state ET and bimolecular $(2,4,5)$ quenching rate constants on $\Delta G_{\mathrm{ET}}{ }^{*}: 1,2$ for radiationless transition ((7); $\left.V_{\mathrm{AD}}=0.01 \mathrm{eV}, h \nu_{V}=0.2 \mathrm{eV}, S=2, \sigma=0.2 \mathrm{eV}\right) ; 3,4$ for Marcus ((2), (3); $\lambda=1.8 \mathrm{eV})$ mechanisms in loose pairs of reactant molecules and 5 for concatenated mechanism in contact pairs ((16); $\left.\log \tau_{0}^{\prime}+a / 2.3 R T=-7.3, b=0, c=0.2 \mathrm{eV}\right)$.

excited molecules $\left(k_{\mathrm{ISC}}^{\prime} / k_{\mathrm{IC}}^{\prime}>k_{\mathrm{ISC}} / k_{\mathrm{IC}}\right)$ the quenching of singlet excited state will increase the triplet yield

$$
\varphi_{T}=k_{\mathrm{ISC}}^{\prime} \tau_{0}^{\prime}\left(1-\frac{\varphi_{F}}{\varphi_{F} 0}\right)+k_{\mathrm{ISC}} \tau_{0}\left(\frac{\varphi_{F}}{\varphi_{F} 0}\right) .
$$

(7) The dependence of apparent activation free energy $\Delta G_{\mathrm{Q}}^{\ddagger}=R T \ln \left(1 / k_{\mathrm{Q}}-1 / k_{\text {Diff }}\right)$ on $\Delta G_{\mathrm{ET}} *$ frequently does not follow either (3) or $(5)$ and has rather low slope $(\ll 1)$ at $\Delta G_{\mathrm{ET}}{ }^{*}>0.3 \mathrm{eV}$ (see several examples in Figures 8-10, 13). This occurs because of the different kinds of the dependences of $\Delta G_{\mathrm{Ex}}{ }^{*}$ and $\Delta G_{\mathrm{Q}}^{\neq}$on $\Delta G_{\mathrm{ET}}{ }^{*}$ : the first (15) is hyperbolic, Marcus (2) is parabolic and Weller's (5) is hyperbolic, but has the opposite orientation.

All these features of the transient exciplex mechanism can be used as criteria for discrimination of ET mechanisms in the particular systems.

Figure 19 shows the dependences of the rate constants of unimolecular and bimolecular reactions of electron transfer on $\Delta G_{\mathrm{ET}}{ }^{*}$ for the three mechanisms: preliminary medium reorganization, radiationless transition and concatenated mechanism. It is seen that concatenated mechanism dominates at $\Delta G_{\mathrm{ET}}{ }^{*}>-(0.3-0.5) \mathrm{eV}$ and the radiationless mechanism-in the range $-3<\Delta G_{\mathrm{ET}}{ }^{*}<-0.5 \mathrm{eV}$. The mechanism of preliminary medium reorganization for bimolecular reactions appeared to be hidden under a diffusional limit.

\section{Conclusion}

Experimental data, discussed here, demonstrate that many bimolecular excited-state ET reactions of organic molecules (at least outside of the diffusion controlled region) follow 
contact two-step mechanism, which involves simultaneous charge shift and medium and reactants' reorganization (stimulated by the strong electronic coupling in the contact pair) and the formation of an intermediate exciplex (with partial charge transfer in the case of excited-state ET), rather than the ordinary one-step long-range mechanism. This adiabatic contact mechanism of ET has a fundamentally different physical behavior than the common diabatic long-range mechanism. In this contact mechanism strong electronic coupling affects the shape of the reaction potential surface and the energy of the transient formed, rather than only a probability of the transition in the diabatic mechanism. This strong electronic coupling causes the appearance of an energy well with lower barriers between the stable states than that found for the transition state of the single-step mechanism. Actually a gradual charge shift can occur already during the approach of the reactant molecules close to each other, and some transient with partial charge transfer can be formed. Under particular conditions activation barriers for such shift can be lower than for electron jump in loose pair of reactant molecules, and this stepwise mechanism can be faster than one-step direct ET and dominates.

The general idea of Marcus on the importance of the medium and reactants' reorganization is still valid for aspects of this multistep mechanism, but in this mechanism strong electronic coupling in the contact reactant pair generates a driving force for the reorganization and eliminates the necessity of thermally activated preliminary reorganization, supposed by the original Marcus mechanism.

The rather long lifetime of the transient intermediate (up to several ns) provides the possibility for various chemical reactions (bond dissociation, isomerization, proton transfer, etc.) as well as radiationless decay processes to compete with the formation of radical ions and significantly reduce the ET quantum yields. The conversion of the transient exciplex into SSRIP and free solvated radical ions requires substantial activation energy related to the medium reorganization (ca. $0.2-0.3 \mathrm{eV}[27,28])$. The concatenated mechanism provides more tools for controlling the rates of ET reactions and yields of the products, affecting several parameters of the transient formed $\left(V_{\mathrm{AD}}, \Delta G_{\mathrm{Ex}}{ }^{*}, \tau_{0}^{\prime}\right)$ by the reactants' structure and medium properties.

The transient exciplex formation (concatenated) mechanism provides an adequate description of the physical behavior of the excited-state electron transfer and the main features of these reactions. The most important peculiarities of this mechanism are (i) a very low activation energy for transient formation and (ii) a substantial competition of various decay processes with the formation of final electron transfer products. Experimental data show that this mechanism dominates for endergonic excited-state electron transfer reactions. The radiationless transition mechanism in loose pairs of reactants dominates for strongly exergonic excited-state electron transfer reactions; however the Marcus mechanism appears to be hidden in the diffusion limit for weakly exergonic excited-state electron transfer reactions.

\section{Appendix}

\section{Energy Diagrams for Exciplex Formation and Decay}

Model energy diagrams for exciplex formation, dissociation into solvent separated radical ion pair and free radical ions (Figures 2, 5, 6) were drawn using common approximation for energy of the composite state

$$
U=U_{\mathrm{LJ}}+U_{\mathrm{Diff}}+\frac{\left(U_{1}+U_{2}\right)}{2}-\left(\frac{\left(U_{1}-U_{2}\right)^{2}}{4}+V_{\mathrm{AD}}{ }^{2}\right)^{1 / 2},
$$

where $U_{1}=\lambda s^{2}, U_{2}=\lambda(1-s)^{2}$, reorganization energy $\lambda=1.44\left(1 / 0.3-1 / r_{\mathrm{AD}}\right)\left(1 / n^{2}-1 / \varepsilon\right), n^{2}=2, \varepsilon=37, s$ is the medium reorganization coordinate, electronic coupling $V_{\mathrm{AD}}=V_{\mathrm{AD}}^{0} \exp \left(r_{\mathrm{AD}} / a\right), V_{\mathrm{AD}}^{0}=1.3 \mathrm{eV}, a=0.2 \mathrm{~nm}$. Short distance molecular repulsion was taken into account as Lennard-Jones potential $U_{\mathrm{LJ}}=0.045\left[\left(0.35 / r_{\mathrm{AD}}\right)^{12}-\right.$ $\left.\left(0.35 / r_{\mathrm{AD}}\right)^{6}\right] \mathrm{eV}$. Activation barriers for diffusion were taken into account in the form of $U_{\text {Diff }}=\Delta G_{\text {Diff }}^{\ddagger}(1+$ $\left.\cos \left(r_{\mathrm{AD}} / 0.11\right)\right) / 2$, where $\Delta G_{\text {Diff }}^{\ddagger}$ was estimated as $\Delta G_{\text {Diff }}^{\ddagger}=$ $2.3 R T\left(\log \left(k_{\mathrm{B}} T / h\right)-k_{\text {Diff }}\right)=0.08 \mathrm{eV}$, using $k_{\text {Diff }}=$ $8 R T / 3000 \eta \approx 2 \times 10^{10} \mathrm{M}^{-1} s^{-1}$ and $k_{\text {Sep }}=(8 R T / 3000 \eta)$ $\left(z_{1} z_{2} e^{2} / \rho \varepsilon k_{\mathrm{B}} T\right) /\left[\exp \left(z_{1} z_{2} e^{2} / \rho \varepsilon k_{\mathrm{B}} T\right)-1\right] \approx 3 \times 10^{10} \mathrm{~s}^{-1}$ for uncharged molecules $\left(\mathrm{A}^{*}\right.$ and $\left.\mathrm{D}\right)$ and ions $\left(\mathrm{A}^{-\bullet}\right.$ and $\left.\mathrm{D}^{+\bullet}\right)$, resp. (where $\eta$ and $\varepsilon$ are the solvent viscosity and dielectric permittivity, resp. $z_{1} e$ and $z_{2} e$ are ion charges, and $\rho$ is the ion radius). This corresponds to activation energy of diffusion $0.16 \mathrm{eV}$ and minima at 0.33 and $1 \mathrm{~nm}$.

Energy diagram in Figure 2 is drawn, using $\Delta G_{\text {Diff }}^{\ddagger}=$ $0.14 \mathrm{eV}, \Delta G_{\mathrm{M}}^{\ddagger}=0.45 \mathrm{eV}$, and $\Delta G_{\mathrm{Ex}}{ }^{*}=-0.15 \mathrm{eV}$, and experimental data on $\Delta G_{1}^{\ddagger}, \Delta G_{\mathrm{Ex}}{ }^{*}$, and $\Delta G_{\mathrm{Ex}}^{\ddagger}$, obtained in [15], and $\Delta G_{\text {CRIP } \rightarrow \text { SSRIP }}^{\ddagger}$ and $\Delta G_{\text {SSRIP } \rightarrow \text { FRI }}^{\ddagger}$, obtained in $[27,28]$.

\section{Acknowledgment}

This work was supported by the Russian Foundation for Basic Research, Project no. 10-03-00486.

\section{References}

[1] R. A. Marcus, "Electron transfer reactions in chemistry. Theory and experiment," Pure and Applied Chemistry, vol. 69, no. 1, pp. 13-29, 1997.

[2] R. A. Marcus and N. Sutin, "Electron transfers in chemistry and biology," Biochimica et Biophysica Acta, vol. 811, no. 3, pp. 265-322, 1985.

[3] A. V. Barzykin, P. A. Frantsuzov, K. Seki, and M. Tachiya, "Solvent effects in nonadiabatic electron-transfer reactions: theoretical aspects," in Advances in Chemical Physics, S. A. Rice, Ed., vol. 123, pp. 511-616, John Wiley \& Sons, New York, NY, USA, 2002.

[4] A. I. Burshtein, "Non-Markovian theories of transfer reactions in luminescence chemiluminescence and photo- and electrochemistry," in Advances in Chemical Physics, S. A. Rice, Ed., vol. 129, pp. 105-418, John Wiley \& Sons, New York, NY, USA, 2004. 
[5] R. A. Marcus, "On the theory of oxidation-reduction reactions involving electron transfer," Journal of Chemical Physics, vol. 24, no. 5, pp. 966-978, 1956.

[6] R. Bolton and M. D. Archer, "Basic electron transfer theory," in Electron Transfer in Inorganic, Organic and Biological Systems, J. R. Bolton, N. Mataga, and G. McLendon, Eds., vol. 228 of Advances in Chemistry, pp. 7-23, 1991.

[7] V. G. Levich and R. R. Dogonadze, "Theory of radiationless electron transitions between ions in solution," Doklady Academii Nauk SSSR, vol. 124, no. 1, pp. 123-126, 1959, (English translation: Academia Nauk SSSR. Proceedings Physical Chemistry Section, vol. 124, no. 1, pp. 9-11, 1959).

[8] J. J. Hopfield, "Electron transfer between biological molecules by thermally activated tunneling," Proceedings of the National Academy of Sciences of the United States of America, vol. 71, no. 9, pp. 3640-3644, 1974.

[9] N. R. Kestner, J. Logan, and J. Jortner, "Thermal electron transfer reactions in polar solvents," Journal of Physical Chemistry, vol. 78, no. 21, pp. 2148-2166, 1974.

[10] M. G. Kuzmin, "Exciplex mechanism of fluorescence quenching in polar media," Pure and Applied Chemistry, vol. 65, no. 8, pp. 1653-1658, 1993.

[11] M. G. Kuzmin, "Exciplex mechanism of excited state electron transfer reactions in polar media," Journal of Photochemistry and Photobiology A, vol. 102, no. 1, pp. 51-57, 1996.

[12] V. N. Grosso, C. A. Chesta, and C. M. Previtali, "Evidence for nonemissive exciplexes in the singlet quenching of polycyclic aromatic hydrocarbons by polychlorobenzenes in cyclohexane," Journal of Photochemistry and Photobiology A, vol. 118, no. 3, pp. 157-163, 1998.

[13] P. Jacques, X. Allonas, M. von Raumer, P. Suppan, and E. Haselbach, "Quenching of triplet benzophenone by methyl and methoxy benzenes: are triplet exciplexes involved?" Journal of Photochemistry and Photobiology A, vol. 111, no. 1-3, pp. 41-45, 1997.

[14] S. M. Hubig and J. K. Kochi, "Electron-transfer mechanisms with photoactivated quinones. The encounter complex versus the Rehm-Weller paradigm," Journal of the American Chemical Society, vol. 121, no. 8, pp. 1688-1694, 1999.

[15] M. G. Kuzmin, I. V. Soboleva, E. V. Dolotova, and D. N. Dogadkin, "Evidence for diffusion-controlled electron transfer in exciplex formation reactions. Medium reorganisation stimulated by strong electronic coupling," Photochemical and Photobiological Sciences, vol. 2, no. 9, pp. 967-974, 2003.

[16] M. G. Kuzmin, I. V. Soboleva, and E. V. Dolotova, "Competition of concatenated and thermally activated medium reorganization in photoinduced electron transfer reactions," High Energy Chemistry, vol. 40, no. 4, pp. 234-247, 2006.

[17] S. Murata and M. Tachiya, "Unified interpretation of exciplex formation and Marcus electron transfer on the basis of two-dimensional free energy surfaces," Journal of Physical Chemistry A, vol. 111, no. 38, pp. 9240-9248, 2007.

[18] H. Knibbe, D. Rehm, and A. Weller, "Bildung von Molekulkomlexen im angeregten Zustand-Zusammanhang zwischen Emissionsmaximum und Reduktionspotential des Elektronakzeptors," Zeitschrift für Physikalische Chemie, vol. 56, no. 1, pp. 95-98, 1967.

[19] H. Beens, H. Knibbe, and A. Weller, "Dipolar nature of molecular complexes formed in the excited state," Journal of Chemical Physics, vol. 47, no. 3, pp. 1183-1184, 1967.

[20] N. Mataga, T. Okada, and N. Yamamoto, "Electronic processes in hetero-excimers and the mechanism of fluorescence quenching," Chemical Physics Letters, vol. 1, no. 1, pp. 119$121,1967$.
[21] M. G. Kuzmin and L. N. Guseva, "Donor-acceptor complexes of singlet excited states of aromatic hydrocarbons with aliphatic amines," Chemical Physics Letters, vol. 3, no. 1, pp. 71-72, 1969.

[22] M. Gordon and W. R. Ware, Eds., The Exciplex, Academic Press, New York, NY, USA, 1975.

[23] M. G. Kuzmin and L. N. Guseva, "Radiationless deactivation in electron transfer reactions," Academia Nauk SSSR. Proceedings Physical Chemistry Section, vol. 200, no. 2, pp. 779-782, 1971.

[24] A. Weller, "Mechanisms of electron-transfer reactions with excited molecules," in Fast Reactions and Primary Processes in Chemical Kinetics, Proceedings of the 5th Nobel Symposium, pp. 413-436, Interscience, 1967.

[25] A. Weller, "Electron-transfer and complex formation in the excited state," Pure and Applied Chemistry, vol. 16, no. 1, pp. 115-124, 1968.

[26] N. Mataga, T. Okada, and K. Ezumi, "Fluorescence of pyrene-N,N-dimethylaniline complex in non-polar solvent," Molecular Physics, vol. 10, no. 2, pp. 203-204, 1966.

[27] B. R. Arnold, D. Noukakis, S. Farid, J. L. Goodman, and I. R. Gould, "Dynamics of interconversion of contact and solventseparated radical-ion pairs," Journal of the American Chemical Society, vol. 117, no. 15, pp. 4399-4400, 1995.

[28] B. R. Arnold, S. Farid, J. L. Goodman, and I. R. Gould, "Absolute energies of interconverting contact and solventseparated radical-ion pairs," Journal of the American Chemical Society, vol. 118, no. 23, pp. 5482-5483, 1996.

[29] M. G. Kuzmin, I. V. Soboleva, E. V. Dolotova, and D. N. Dogadkin, "The nature of internal conversion and intersystem crossing in exciplexes," High Energy Chemistry, vol. 39, no. 2, pp. 86-96, 2005.

[30] J. R. Miller, "Puzzles of electron transfer," in Electron Transfer in Inorganic, Organic and Biological Systems, J. R. Bolton, N. Mataga, and G. McLendon, Eds., vol. 228 of Advances in Chemistry, pp. 265-276, 1991.

[31] D. Rehm and A. Weller, "Kinetics of fluoresecence quenching by electron and H-atom transfer," Israel Journal of Chemistry, vol. 8, no. 2, pp. 259-271, 1970.

[32] M. G. Kuzmin, I. V. Soboleva, and E. V. Dolotova, "The behavior of exciplex decay processes and interplay of radiationless transition and preliminary reorganization mechanisms of electron transfer in loose and tight pairs of reactants," Journal of Physical Chemistry A, vol. 111, no. 2, pp. 206-215, 2007.

[33] J. Gersdorf, J. Mattay, and H. Görner, "Radical cations. 3. Photoreactions of biacetyl, benzophenone, and benzil with electron-rich alkenes," Journal of the American Chemical Society, vol. 109, no. 4, pp. 1203-1209, 1987.

[34] M. G. Kuz'min, "Medium polarity effects on the electronic structure emission spectra of exciplexes," Russian Journal of Physical Chemistry A, vol. 73, no. 10, pp. 1625-1632, 1999.

[35] D. N. Dogadkin, I. V. Soboleva, and M. G. Kuzmin, "Effect of solvent polarity on the electronic structure and emission frequencies of exciplexes of aromatic hydrocarbons with methoxybenzenes and methylnaphthalenes," High Energy Chemistry, vol. 35, no. 2, pp. 107-114, 2001.

[36] D. N. Dogadkin, I. V. Soboleva, and M. G. Kuz'min, "Formation enthalpy and entropy of exciplexes with variable extent of charge transfer in solvents of different polarity," High Energy Chemistry, vol. 35, no. 4, pp. 251-257, 2001.

[37] M. G. Kuz'min, E. V. Dolotova, and I. V. Soboleva, "The influence of solvation on the electronic structure, emission 
spectra, and thermodynamic characteristics of formation of exciplexes," Russian Journal of Physical Chemistry A, vol. 76, no. 7, pp. 1109-1118, 2002.

[38] D. N. Dogadkin, I. V. Soboleva, and M. G. Kuz'min, "Formation enthalpy of exciplexes with partial charge transfer as a function of the electron-transfer driving force," High Energy Chemistry, vol. 36, no. 6, pp. 383-390, 2002.

[39] P. Jacques, X. Allonas, P. Suppan, and M. von Raumer, "The interplay between steric hindrance and exergonicity in the rates of excited state quenching," Journal of Photochemistry and Photobiology A, vol. 101, no. 2-3, pp. 183-184, 1996.

[40] M. Dossot, D. Burget, X. Allonas, and P. Jacques, "From RehmWeller to exciplex mechanisms by a structural effect: fluorescence quenching of a thioxanthone derivative by methoxyand methyl-substituted benzenes in acetonitrile," New Journal of Chemistry, vol. 25, no. 2, pp. 194-196, 2001.

[41] M. Dossot, X. Allonas, and P. Jacques, "Singlet exciplexes between a thioxanthone derivative and substituted aromatic quenchers: role of the resonance integral," Chemistry A, vol. 11, no. 6, pp. 1763-1770, 2005.

[42] J. Mattay, J. Gersdorf, and K. Buchkremer, "Photoreactions of biacetyl with electron-rich olefins. An extended mechanism," Chemische Berichte, vol. 120, no. 3, pp. 307-318, 1987.

[43] R. E. Föll, H. E. A. Kramer, and U. E. Steiner, "Role of charge transfer and spin-orbit coupling in fluorescence quenching. A case study with oxonine and substituted benzenes," Journal of Physical Chemistry, vol. 94, no. 6, pp. 2476-2487, 1990.

[44] E. V. Dolotova, I. V. Soboleva, and M. G. Kuz'min, "Activation enthalpy and entropy for the decay of 9-cyanophenanthrene exciplexes," High Energy Chemistry, vol. 37, no. 4, pp. 231-240, 2003.

[45] D. N. Dogadkin, I. V. Soboleva, and M. G. Kuz'min, “Activation parameters for the formation and decay of partial-chargetransfer exciplexes of 9-cyanoanthracene, 1,12-benzperylene, and pyrene," High Energy Chemistry, vol. 38, no. 2, pp. 108114, 2004.

[46] D. N. Dogadkin, E. V. Dolotova, I. V. Soboleva et al., "Mechanism of exciplex decay: the quantum yields and the rate constants of triplet formation from 9-cyanophenanthrene exciplexes," High Energy Chemistry, vol. 38, no. 6, pp. 386-391, 2004.

[47] D. N. Dogadkin, E. V. Dolotova, I. V. Soboleva et al., "Mechanism of exciplex decay: the quantum yields and the rate constants of radical ion formation from exciplexes with partial charge transfer," High Energy Chemistry, vol. 38, no. 6, pp. 392-400, 2004.

[48] Y. Wang, O. Haze, J. P. Dinnocenzo, S. Farid, R. S. Farid, and I. R. Gould, "Bonded exciplexes. A new concept in photochemical reactions," Journal of Organic Chemistry, vol. 72, no. 18, pp. 6970-6981, 2007.

[49] M. G. Kuzmin and N. A. Sadovskii, "Study of the kinetics of fast reactions of excited molecules by nanosecond-pulse fluorometry," High Energy Chemistry, vol. 9, no. 4, pp. 255270, 1975.

[50] H. Miyasaka, A. Tabata, K. Kamada, and N. Mataga, "Femtosecond-picosecond laser photolysis studies on the mechanisms of electron transfer induced by hydrogenbonding interactions in nonpolar solutions: 1-aminopyrenepyridine systems," Journal of the American Chemical Society, vol. 115, no. 16, pp. 7335-7342, 1993.

[51] N. Mataga and H. Miyasaka, "Photoinduced charge transfer phenomena: femtosecond-picosecond laser photolysis studies," Progress in Reaction Kinetics, vol. 19, no. 4, pp. 317-430, 1994.
[52] N. Mataga, "Development of exciplex chemistry: some fundamental aspects," Pure and Applied Chemistry, vol. 69, no. 4, pp. 729-734, 1997.

[53] E. Teller, "The crossing of potential surfaces," Journal of Physical Chemistry, vol. 41, no. 1, pp. 109-116, 1937.

[54] A. Bjerre and E. E. Nikitin, "Energy transfer in collisions of an excited sodium atom with a nitrogen molecule," Chemical Physics Letters, vol. 1, no. 5, pp. 179-181, 1967.

[55] E. E. Nikitin, "Non-adiabatic energy transfer in gases," in Fast Reactions and Primary Processes in Chemical Kinetics, S. Claesson, Ed., Proceedings of 5th Nobel Symposium, pp. 165183, Interscience, New York, YN, USA, 1967.

[56] J. Michl, "Energy barriers in photochemical reactions. A case for the relevance of Woodward-Hoffmann-type correlations," Journal of the American Chemical Society, vol. 93, no. 2, pp. 523-524, 1971.

[57] J. Michl, "Photochemical reactions of large molecules. I. A simple physical model of photochemical reactivity," Molecular Photochemistry, vol. 4, no. 2, pp. 243-255, 1972.

[58] J. Michl, "Photochemical reactions of large molecules. II. application of the model to organic photochemistry," Molecular Photochemistry, vol. 4, no. 2, pp. 257-286, 1972.

[59] J. Michl, "Photochemical reactions of large molecules. III. Use of correlation diagrams for prediction of energy barriers," Molecular Photochemistry, vol. 4, no. 2, pp. 287-314, 1972.

[60] J. Michl, "Model calculations of photochemical reactivity," Pure and Applied Chemistry, vol. 41, no. 4, pp. 507-534, 1975.

[61] M. Klessinger and J. Michl, Excited States and Photochemistry of Organic Molecules, Wiley-VCH, New York, NY, USA, 1995.

[62] M. A. Robb, F. Bernardi, and M. Olivucci, "Conical intersections as a mechanistic feature of organic photochemistry," Pure and Applied Chemistry, vol. 67, no. 5, pp. 783-789, 1995.

[63] M. Z. Zgierski, T. Fujiwara, and E. C. Lim, "Conical intersections and ultrafast intramolecular excited-state dynamics in nucleic acid bases and electron donor-acceptor molecules," Chemical Physics Letters, vol. 463, no. 4-6, pp. 289-299, 2008.

[64] K. Kikuchi, Y. Takahashi, T. Katagiri, T. Niwa, M. Hoshi, and T. Miyashi, "A critical consideration on the lack of inverted region in the Rehm-Weller plot for electron-transfer fluorescence quenching," Chemical Physics Letters, vol. 180, no. 5, pp. 403-408, 1991.

[65] K. Kikuchi, Y. Takahashi, M. Hoshi, T. Niwa, T. Katagiri, and T. Miyashi, "Free enthalpy dependence of free-radical yield of photoinduced electron transfer in acetonitrile," Journal of Physical Chemistry, vol. 93, no. 6, pp. 2378-2381, 1991.

[66] E. Dolotova, D. Dogadkin, I. Soboleva, M. Kuzmin, O. Nicolet, and E. Vauthey, "Lifetimes of partial charge transfer exciplexes of 9-cyanophenanthrene and 9-cyanoanthracene," Chemical Physics Letters, vol. 380, no. 5-6, pp. 729-735, 2003.

[67] C. M. Previtali, "Solvent effects on intermolecular electron transfer processes," Pure and Applied Chemistry, vol. 67, no. 1, pp. 127-132, 1995.

[68] M. G. Kuzmin, N. A. Sadovskii, J. A. Weinstein, and O. M. Soloveychik, "Influence of exciplex formation in polar media on fluorescence quenching mechanisms," High Energy Chemistry, vol. 26, no. 6, pp. 416-421, 1992.

[69] Y. A. Weinstein, N. A. Sadovskii, and M. G. Kuzmin, "Fluorescence quenching of pyrene by weak electron donors and acceptors in polar media: the role of exciplexes in the formation of triplet states and radical ions," High Energy Chemistry, vol. 28, no. 3, pp. 211-218, 1994.

[70] N. A. Sadovskii, O. I. Kutsenok, Y. A. Weinstein, and M. G. Kuz'min, "Spectral properties and electronic structure of 
exciplexes of aromatic compounds in acetonitrile," Russian Journal of Physical Chemistry A, vol. 70, no. 11, pp. 1861-1866, 1996.

[71] M. G. Kuzmin, I. V. Soboleva, and E. V. Dolotova, "Evolution of the reaction mechanism during ultrafast photoinduced electron transfer," Journal of Physical Chemistry A, vol. 112, no. 23, pp. 5131-5137, 2008.

[72] I. R. Gould, R. H. Young, L. J. Mueller, A. C. Albrecbt, and S. Farid, "Electronic structures of exciplexes and excited chargetransfer complexes," Journal of the American Chemical Society, vol. 116, no. 18, pp. 8188-8199, 1994.

[73] A. Weller, "Photoinduced electron-transfer in solutionexciplex and radical ion-pair formation free enthalpies and their solvent dependence," Zeitschrift für Physikalische Chemie, vol. 133, no. 1, pp. 93-98, 1982.

[74] N. A. Sadovskii, R. D. Shilling, and M. G. Kuzmin, "Quenching of excimers by electron donors," Journal of Photochemistry, vol. 31, no. 2-3, pp. 247-252, 1985.

[75] I. V. Soboleva, N. A. Sadovskii, and M. G. Kuzmin, "Effect of the nature of the donor on the intersystem crossing in 9,10dicyanoanthracene exciplexes in heptane," Academia Nauk SSSR. Proceedings. Physical Chemistry Section, vol. 238, no. 1, pp. 70-73, 1978. 


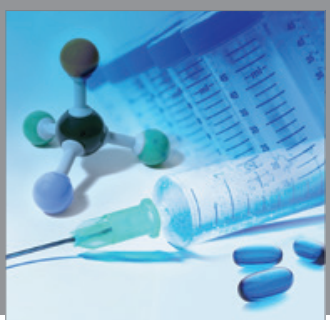

International Journal of

Medicinal Chemistry

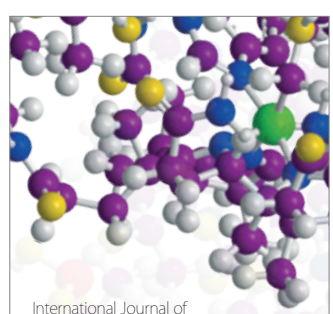

Carbohydrate Chemistry

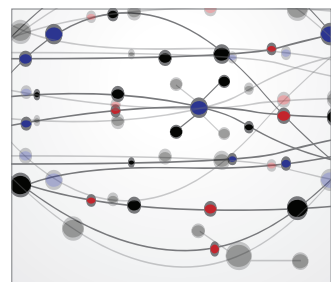

The Scientific World Journal
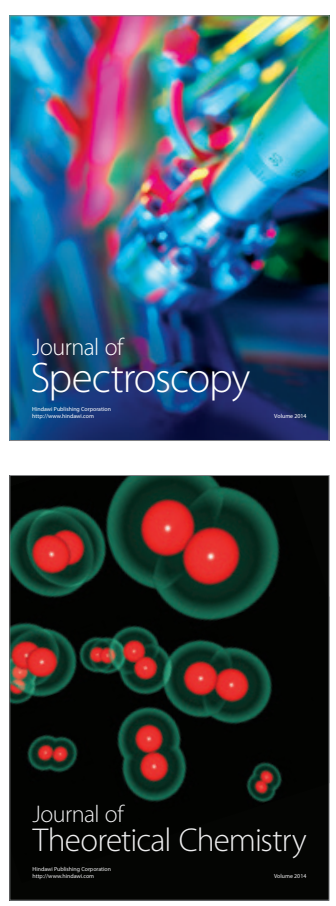
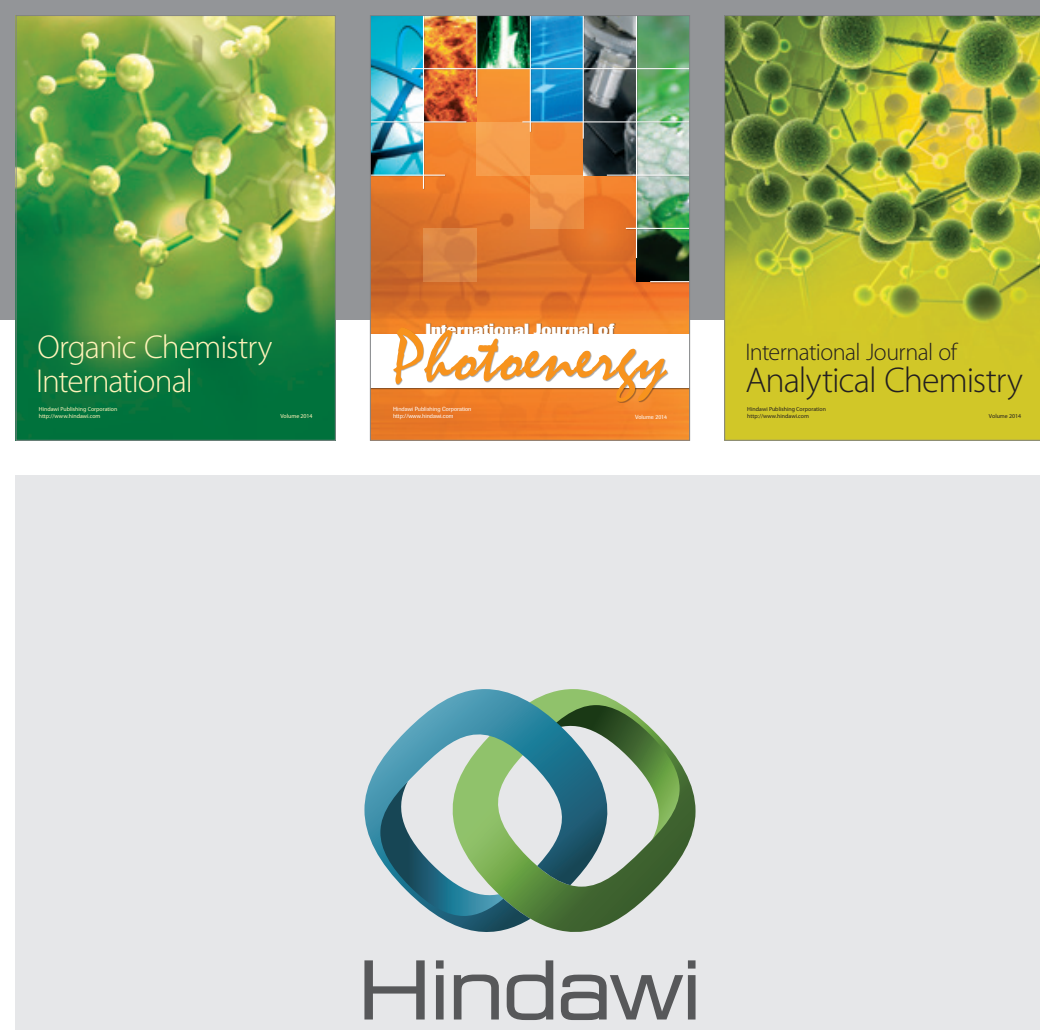

Submit your manuscripts at

http://www.hindawi.com
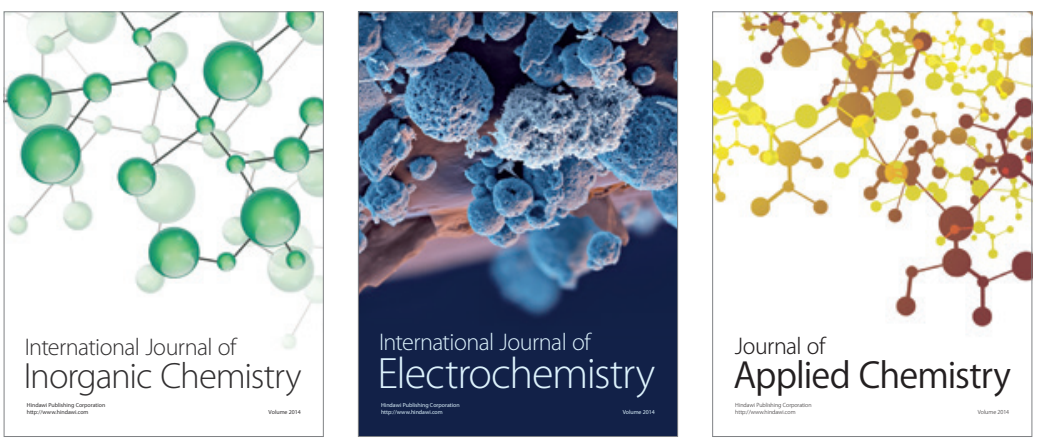

Journal of

Applied Chemistry
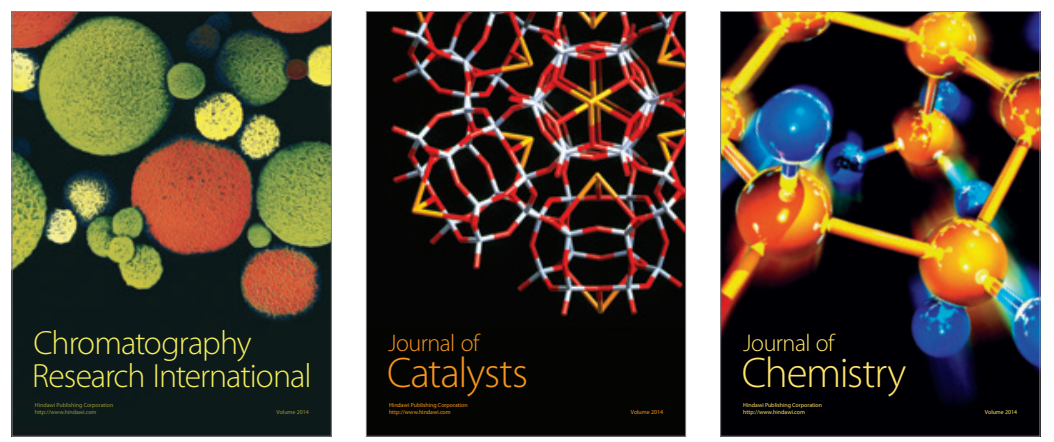
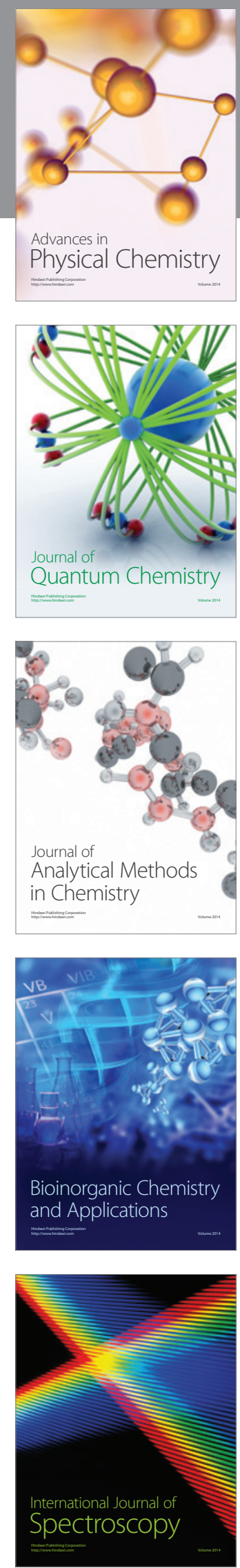